\title{
Developing a Social Evolutionary Measure of Child and Adolescent Hedonic and Eudaimonic Wellbeing in Rural Sierra Leone
}

\author{
Jennifer E. Symonds ${ }^{1}$ (D) Seaneen Sloan ${ }^{1} \cdot$ Michelle Kearns $^{1} \cdot$ Dympna Devine $^{1}$. \\ Ciaran Sugrue $^{1} \cdot$ Sachita Suryanaryan $^{1} \cdot$ Daniel Capistrano $^{1} \cdot$ Elena Samonova $^{1}$
}

Accepted: 17 September 2021 / Published online: 12 October 2021

(C) The Author(s) 2021

\begin{abstract}
The study establishes the validity of a new measure of child and adolescent hedonic and eudaimonic wellbeing designed for international use and based on social evolutionary theory. The measure advances the fields of psychometrics and happiness studies by providing the first a-contextual assessment of both hedonic and eudaimonic wellbeing that is suitable for use with children and adolescents. The Child and Adolescent Personal and Social Assessment of Wellbeing contains a brief measure (8-items) of children's global hedonia and eudaimonia, and domain specific measures (8-items each) of children's wellbeing in relation to peers, teachers, and families. To develop the measure, we conducted a mixed methods investigation in rural Sierra Leone. We researched the ecological validity of the conceptual framework in a qualitative study of 40 participants (19 children and 21 adults) living in two communities. We developed the quantitative items through a two-phase pilot study of 200 children living in ten communities. We examined the psychometric properties of the measure in the pilot study data, and in a larger dataset generated with 2324 children living in 95 communities. The qualitative results showed that the participants experienced the framework components (e.g., social acceptance) in their daily lives, and that each component was intertwined with indigenous perspectives on child wellbeing. The quantitative results demonstrated that the measure had good dimensional, concurrent, and predictive validity.
\end{abstract}

Keywords Africa $\cdot$ Childhood $\cdot$ Low-income $\cdot$ Measure $\cdot$ Social evolution $\cdot$ Wellbeing

\section{Introduction}

Reviews of measuring children's wellbeing have revealed that in low-income countries (Ager et al., 2014) and high-income countries (Tsang et al., 2012) wellbeing is often conceptualized and measured using a diverse set of positive psychology constructs, for

Jennifer E. Symonds

jennifer.symonds@ucd.ie

1 School of Education, University College Dublin, Belfield, Dublin 4, Ireland 
example self-esteem, hope, emotional regulation and grit. The diversity of these concepts and scales makes it challenging for researchers to carry out reviews or meta-analyzes of wellbeing measures. It also inhibits our progress in building an integrated hedonic and eudaimonic perspective on children's wellbeing in low-income countries. Although a few studies do measure children's subjective wellbeing in low-income countries, for example the International Survey of Children's Wellbeing (ISCIWeb) (Rees \& Main, 2015), there is no psychometric measure that is specifically designed to capture both hedonic and eudaimonic wellbeing that has been developed for use with children in these contexts.

To facilitate a randomized controlled trial of an intervention to promote children's literacy and wellbeing in rural Sierra Leone, West Africa, we searched for a psychometric measure of children's psychological wellbeing. Following our theoretical perspective on wellbeing, we wanted the measure to capture functional indicators of both hedonic and eudaimonic wellbeing that would make sense in the cultural context and elsewhere. After our systematic search revealed that there was no existing measure fitting with this perspective, we designed and tested a measure of children's hedonic and eudaimonic wellbeing in a longitudinal study of children. The resultant measure can be used in future research for wellbeing assessment and should also have utility for the design and execution of future interventions for promoting children's wellbeing.

In this paper we report on: (1) our systematic search for measures of children's hedonic and eudaimonic wellbeing suitable for administration in low income, humanitarian contexts, (2) our qualitative investigation of indigenous perspectives on hedonic and eudaimonic wellbeing $(\mathrm{N}=40)$ carried out to inform the measure development, (3) a quantitative pilot study $(\mathrm{N}=200)$ where we developed the measure, and (4) a second quantitative study with a larger sample $(\mathrm{N}=2324)$ where we performed extended validity tests of the measure.

\subsection{A Social Evolutionary Conceptualization of Eudaimonia and Hedonia}

In our study we were interested in measuring children's wellbeing in rural Sierra Leone. We foregrounded our conceptualization using the perspectives of eudaimonia (living well or virtuously i.e., doing well) and hedonia (experiencing positive emotion i.e., feeling good) (David et al., 2013). For children to build up their perceptions of doing well and feeling good, they necessarily must refer to their lived experiences, to evaluate how they are feeling and doing. For this reason, we took the approach that children's hedonic and eudaimonic wellbeing must be experienced, or 'lived', through some type of function (e.g., Vitters $\varnothing, 2013)$. Specifically, we were interested in how the fulfilment of basic psychological needs (e.g., Maslow, 1943), of relatedness, competence, and autonomy (Ryan \& Deci, 2019), might facilitate children to perceive they are doing well and feeling good.

The concepts of hedonia, eudaimonia, and the basic needs of autonomy, relatedness, and competence, have been advanced primarily in Western humanistic psychological literature (Reeve, 2015). However, research in Africa also demonstrates their application with nonWestern, indigenous populations. In South Africa, Savahl and colleagues tested the suitability of Western developed measures of hedonic wellbeing (the Personal Wellbeing Index: Cummings \& Lau, 2005) and eudaimonic wellbeing (i.e., the Children's Hope Scale: Snyder et al., 1997) with an adolescent sample. Confirmatory factor analysis found that both measures fit the data well (Savahl et al., 2017, 2020) and factorial invariance testing across higher and lower socioeconomic status groups found that the hedonic measure had scalar equivalence across groups (Savahl et al., 2017). Researchers working in Ghana (Wilson 
Fadiji et al., 2019) also found that Western developed measures of hedonia (the Satisfaction with Life Scale: Diener et al., 1985) and eudaimonia (the Meaning in Life Questionnaire: Steger et al., 2006) were a good fit to data gathered with their adult sample.

Qualitative research in Africa has observed hedonic and eudaimonic aspects of wellbeing being freely described by adults and children. For example, Ghanaian adults have described hedonic aspects of wellbeing (e.g., "life free of stress", p. 20) and eudaimonic aspects of wellbeing (e.g., "to be free to be yourself", p. 18) (Wilson Fadiji et al., 2019). Ghanaian adolescents have also discussed their feelings of confidence in the future (in keeping with notions of eudaimonia) and global evaluations of life (specifically life-satisfaction) (Wilson \& Somhlaba, 2016). Furthermore, in South Africa, children have discussed feeling good about themselves as central to their psychological wellbeing (Savahl et al., 2015).

Research in Africa also supports our assumption that functional wellbeing can be manifest in children's experiences of autonomy, relatedness, and competence. Ethiopian children and adults in the Young Lives study were asked to describe a child who was 'doing well/not doing well' (Crivello et al., 2009). Indicators of child wellbeing included having high quality relationships (i.e., relatedness), having the freedom to attend school and to play (i.e., autonomous functioning in accordance with values), and behaving well (i.e., competence in behaving in accordance with observable social norms). In a qualitative study conducted in rural Zambia (and India), White and Jha (2018) developed a framework of subjective wellbeing that comprised agency and participation, values and meaning (i.e., autonomy), competence and self-worth (i.e., competence), social connections and close relationships (i.e., relatedness) as well as economic confidence, and physical and mental health. Both studies (Crivello et al., 2009; White \& Jha, 2018) indicate that autonomy, relatedness, and competence in African samples are facilitated through specific lived experiences that are aligned with the cultural milieu (e.g., being able to go to school in an environment where access to education can be restricted by a lack of income or competing activities such as manual labor).

To extend on the notion that wellbeing is hedonic and eudaimonic, and comprises functional experiences of relatedness, autonomy, and competence, we took an evolutionary approach to understanding how wellbeing might emerge in children in Africa and in other cultural contexts. This perspective aligns with White's (2015) relational wellbeing conceptualization that describes individual and collective wellbeing as "emergent through the interplay of personal, societal, and environmental processes" (White, 2015, p. 5). In the social evolutionary perspective (Tomasello \& Vaish, 2013), like other mammals, humans have evolved to live in cooperative social groups that are essential to our flourishing as a species. Within those groups, typically functioning individuals help others which increases their own chances of personal fitness, fecundity, and survival, reciprocally sustaining the health of the group (Rodrigues \& Kokko, 2016). Therefore, for the group to thrive there must be a dynamic system of reciprocal helping, operating as a downward influence of care from others, the upward influence of helping others, and inclusion in the system by being accepted as someone who should receive, and can give, help. People who are rejected by their communities are unable to receive help which can be a serious threat to their survival. This creates the conditions for hypercooperative societies (Tomasello \& Vaish, 2013), where our basic needs are shaped and fulfilled in the context of helping others.

In order to help others, people must develop the skills or competencies that are central to the flourishing of that group, such as competencies in providing food, caring for infants, and mitigating physical harm (Kingdon \& Knight, 2006). Although the competencies might differ according to culturally and contextually relevant needs, there should also 
be some consistency in what competencies are important, based on humans' fundamental requirements for fitness, survival and fecundity (i.e., providing food is necessary in all cultures, but competencies for food provision will differ in form between a high-income nation where people rely on supermarkets, and a low-income village in rural Sierra Leone where people typically collect and grow much of their own food). For example, in rural Sierra Leone, being able to provide economic wealth and safety to the family, developing skills in manual labor, and developing competencies in domestic work, are critical to the welfare of society (Devine et al., in press a).

The system of reciprocal helping is guided by a structure of observable social norms (e.g., children should help their parents with domestic and agricultural work) that creates stability in the system (Henrich \& Muthukrishna, 2021). Individual people need autonomy within this structure, so that the social norms can be transformed to promote greater societal flourishing as new ideas and competencies emerge within changing social and environmental contexts. Autonomy in this perspective refers to the freedom to "act in accord with authentic interests or integrated values and desires" (Chirkov et al., 2003, p. 98), which can result in people supporting or rejecting social norms, leading to both stability and change in the system. In the Young Lives Ethiopian sample (Crivello et al., 2009) and in qualitative case studies of rural Sierra Leonian village communities, having the autonomy to go to school was referenced as important for children, who without education were projected to have poorer quality outcomes in terms of future employment (mainly males) and ability to care for the family (mainly females) (Devine et al., in press a, b). Here we can observe the importance of children's autonomy to live their lives in accordance with current cultural values (education and gender norms).

In any complex social and environmental system, challenges to psychological wellbeing occur. To manage these, people need to be resilient to setbacks, so they can find adaptive pathways to help others in the face of challenge and strive to receive the resources they need for fitness, survival, and fecundity in conditions of scarcity. Furthermore, the environment should be balanced in favor of safety, rather than extreme challenge (i.e., threat), so that individuals are not overburdened by acute stress responses and have the necessary energy and competence to help others to promote societal flourishing. Threats perceived by children in Sierra Leone have included lack of food, manual labor, and other types of material deprivation, and these threats are linked to feelings of anger, sadness, worry, fear, and disruptive behaviors (Thulin et al., 2020). In Ethiopia (Crivello et al., 2009) and Ghana (Wilson \& Somhlaba, 2016), children and adolescents have reported similar cultural, social, and nutritional threats to their wellbeing (i.e., death of parents, lack of access to education, early marriage, early entry into the labor force, lack of food, lack of money, disease, and environmental disaster). Psychological resilience to the negative impact of these factors, for example by feeling confident and hopeful in the future (Wilson \& Somhlaba, 2016), social processes that ameliorate these factors such as relational support, and economic resources such as health care services that reduce these threats (White, 2015), are important for maintaining wellbeing in communities.

In summary, eudaimonic wellbeing (doing well) should be promoted when people are able to competently do things that help others (in some manner) within a wider social system, when they have autonomy to act in accordance with their belief system, and when they are able to overcome setbacks so that they can function adaptively. Hedonic wellbeing should be promoted when people feel accepted and cared for by others, and when they feel safe in their environment, so that they can function effectively. Children who are socialized into this system should also experience similar drivers and indicators of wellbeing, although this will be shaped by constructions of childhood that help 
determine the role of children as active agents in society. This conceptualization has led us to consider eight key indicators of wellbeing (Fig. 1): four representing eudaimonia (autonomy, competence, resilience, and helping) and four representing hedonia (care, acceptance, safety, and happiness) — that we used to inform our measure design.

\subsection{Global and Domain Specific Eudaimonia and Hedonia}

As well as identifying eight key indicators of wellbeing for our study, we were also interested in exploring children's hedonia and eudaimonia from a holistic, and multidimensional perspective. First, we were interested in children's overall experiences of wellbeing, i.e., how they rated their eudaimonia and hedonia independently of any one lived experience. This can be understood as global hedonic and eudaimonic wellbeing, i.e., how safe or competent children feel in general. Second, we were also interested in children's experiences of hedonia and eudaimonia with respect to key proximal developmental contexts in their daily lives, i.e., their experiences with peers, teachers, and families. Using this approach, we hoped to capture a summary of children's wellbeing as well as more refined information about wellbeing in key dimensions.

This global and domain specific approach to conceptualizing and measuring psychological constructs has been used often, and usefully, in studies of child development. In 1982, Harter made the point that a child's overarching perceptions of their competence is not simply the sum of their perceptions of competence in different domains (e.g., sports, friendships). Rather, their overarching self-competence is unique, and emerges from their experiences of self-competence across different sets of experiences. This informed the development of Harter's Self-Perception Profile (2012) which measures children's global perceived competence (self-worth), and domain specific perceptions of competence (of scholastic, social, athletic, physical, and behavioral dimensions). More recently, Queiroz et al. (2020) operationalized self-esteem as both global, and domain specific (measured across academic, social, emotional, family, and physical dimensions). Following those approaches, we were interested in investigating children's global and domain specific hedonic and eudaimonic wellbeing, operationalized using key indicators that we have theorized above as having social evolutionary importance.

Psychological Wellbeing

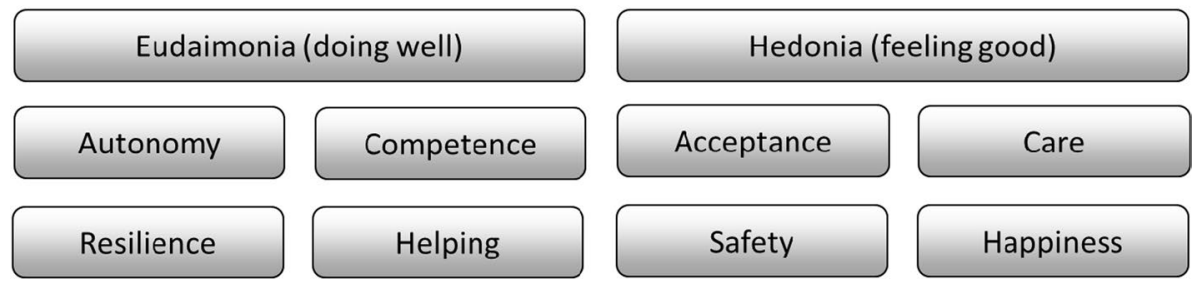

Fig. 1 Social evolutionary conceptualization for measuring child wellbeing 


\subsection{International Transferability and Local Relativity}

Our social evolutionary perspective assumes that key indicators of eudaimonia and hedonia are universalistic-but not universal. Here we follow the cross-cultural psychological perspective of Berry et al. (2008), who posit three key assumptions about how psychology works across cultures. First, in the absolutist perspective, psychological phenomena are closely similar in all cultures (e.g., depression, gratitude), and culture has a minimal role in shaping psychology. Second, in the relativistic perspective, all human psychology and behavior is culturally shaped and individual people and cultures can only be understood in their own terms. Third, in the universalistic perspective, basic psychological processes are common to all people (such as those we outline in our social evolutionary perspective, e.g., happiness); however, culture influences how those processes develop and manifest in individuals and social groups. Universalism is the middle ground between absolutism and cultural relativism and provides a workable solution to transferring assumptions about wellbeing across cultures, whilst recognizing that wellbeing will always be culturally relative. Taking a universalistic perspective, we developed our measure of wellbeing within a specific cultural context whilst testing for the transferability of our assumed universalistic key indicators of eudaimonia (eudaimonia (autonomy, competence, resilience, and helping) and hedonia (care, acceptance, safety, and happiness) in the cultural sample. This resulted in a measure that can theoretically be extended to other cultures whilst acknowledging that there might be cultural relativity in how those indicators are experienced and expressed.

\section{General Research Context}

The research team were commissioned to conduct a randomized controlled trial of an intervention designed to promote children's literacy and wellbeing in rural Sierra Leone, by an international Non-Government Organization (NGO), in early 2017. All research procedures were approved by the university's Human Research Ethics Committee in April 2017. The first year of the project was dedicated to piloting the research methods and the NGO's intervention across a single school year (October 2017-May 2018). During this year we conducted a quantitative pre-test in October 2017, piloted our qualitative methods between November and February, then conducted a quantitative post-test in May 2018. After the pilot results were analyzed, we embarked on the threeyear main trial in October 2018.

The schedule for our development of the wellbeing measure fit into this research timeline. Before entering the field in October 2017, we conducted a systematic review to identify any wellbeing measures that would fit our theoretical perspective and sample. This research is reported in Study 1. Between the quantitative pre- and post-tests, we carried out a qualitative focus group investigation of indigenous perspectives on wellbeing and understandings of our wellbeing theoretical framework. We report on this investigation in Study 2. The qualitative focus group investigation informed the final set of wellbeing measurement items that we piloted in the pilot-study post-test. The final set of items were also informed by our ethnographic study (Devine et al., 2021) where one researcher spent approximately 16 weeks in each of two case study communities interviewing children, their parents and grandparents as well as village elders. The 
quantitative development and testing of the measure took place across the evaluation pilot-study (testing initial items in the pre-test, then testing a refined set of items in the post-test) and in the first wave of the main study. All quantitative testing is reported in Study 3 .

\section{Study 1: Systematic Search for Measures of Children's Wellbeing}

Before we decided to design a new measure, we conducted a systematic literature search for measures of children's hedonic and eudaimonic wellbeing in low income, humanitarian contexts. A previous search by Ager et al. (2014) catalogued 47 different measures of child and adolescent mental health and psychosocial wellbeing in low-income, humanitarian contexts. However, most of these measures targeted very specific types of mental illness and trauma (e.g., anxiety, post-traumatic stress) and mental health (e.g., hope, self-esteem, controlled behavior). Only one measure identified by Ager et al. (2014) included items that captured both hedonic and eudaimonic wellbeing: The Orphans and Vulnerable Children Wellbeing Tool (Senefeld et al., 2009). This 27-item measure taps into hedonia and eudaimonia through items such as I am happy as other kids my age. However, many items in this measure were deemed unsuitable for the current study. Indeed, several were ambiguous in whether they indicated wellbeing or ill-being depending on the cultural context, e.g. My school attendance is affected by my need to work. Furthermore, there were no items in this measure capturing autonomy which is central to our perspective on child wellbeing.

\subsection{Search Methodology}

Using Ager et al.'s (2014) review as a baseline, we conducted an updated systematic search for measures of children's hedonic and eudaimonic wellbeing used in low-income, humanitarian contexts. We searched the Scopus and ERIC databases for research published between January 2013 [the end date of the Ager et al. (2014) review] and July 2017 (the month of the updated search-preceding our longitudinal empirical investigation which began in 2018). The following search terms were fed into the databases: wellbeing OR "well-being” OR "mental health" OR functioning) AND (assessment OR measurement OR instrument OR questionnaire OR survey) AND (child* OR adolesc* OR "young people") AND (humanitarian OR "low-income" OR "developing nation" OR "developing nations" OR "developing count*” OR "Global South" OR NGO OR "non-profit"). Documents meeting inclusion criteria had to be published in English and include a research tool or approach administered with children to assess their psychological functioning in humanitarian situations in low-income countries. The search terms and inclusion/exclusion criteria described above were adapted from Ager et al. (2014).

After duplicates were removed, the search returned a total of 568 records. After title and abstract screening, we removed 398 records that did not meet inclusion criteria. We then screened the remaining 170 records for details of wellbeing measures. This resulted in removing a further 93 records, including reviews published between 2013 and 2017 that covered the same time period as the Ager et al. (2014) review. A total of 77 records remained comprising 5 review papers and 72 empirical studies.

The full texts of the 77 included publications were scanned and each measure identified was entered into an Excel spreadsheet. This resulted in a list of 73 measures. A further 21 measures were added from a hand search of online databases conducted in July 
2017, covering Young Lives, Oxfam, UNICEF, Save the Children, WHO, Psychosocial Working Group, Research for Health in Humanitarian Crises, International Survey of Children's Wellbeing (ISCIWeb), and Relief Web. Finally, the 46 measures identified by Ager et al. (2014) were added to the database. After duplicate measures were removed, including revised and brief versions of the same measure, 95 measures remained.

Next, the researchers reviewed the 95 measures to identify those that captured children's wellbeing as a multidimensional construct (e.g., combining social, personal, and emotional functioning), or focused on a global form of hedonia or eudaimonia (e.g., life satisfaction). This ruled out measures of negative constructs (e.g., anxiety, depression, behavioral problems, and post-traumatic stress) or single positive psychological constructs (e.g., grit, optimism) that did not encapsulate wellbeing as a global or a domain specific construct broad enough to represent hedonia or eudaimonia. Out of the 18 measures that met these narrower inclusion criteria, 11 were freely available (Table 1), 5 were privatized, 1 was in a copyrighted book, and 2 could not be located.

\subsection{Results}

The systematic search process uncovered 11 freely available measures detailed in Table 1. These measures were reviewed for their suitability for (a) capturing child wellbeing in line with our social evolutionary conceptualization and (b) feasibility of administration in a larger study where we also measured children's literacy (not reported here). We extracted the 262 items from the measures and coded each item for (a) its personal, social, or environmental context (e.g., self, peers, family, teachers, community, school, neighborhood, etc.), and (b) whether it tapped into any of our eight wellbeing indicators (Fig. 1). Only one measure-the Young Lives' questionnaire for 8-year-olds (younger cohort) — covered all eight indicators. However, the 54-item questionnaire was too long for administration alongside our literacy measure. Furthermore, it was not a psychometric measure of hedonia and eudaimonia. After completing this final step of our search for a suitable measure, we concluded that there was a legitimate need to design a psychometric measure of children's hedonic and eudaimonic wellbeing for use in low income, humanitarian contexts.

\section{Study 2: Qualitative Investigation of Wellbeing}

To inform the measure design we conducted a qualitative investigation that aimed to (1) uncover indigenous perspective on child wellbeing and (2) explore the meaning of the four indicators of eudaimonia (autonomy, competence, resilience, and helping) and four indicators of hedonia (care, acceptance, safety, and happiness), as developed in our theoretical perspective, to local adults and children. This first empirical study was designed in line with Berry et al.'s (2008) first two goals of cross-cultural psychology: the discovery goal (to uncover cultural definitions of wellbeing) and the transport and test goal (to test our social evolutionary perspective in an indigenous culture). As reported in the research context section, the qualitative study received ethical approval from the university's Human Research Ethics Committee. 


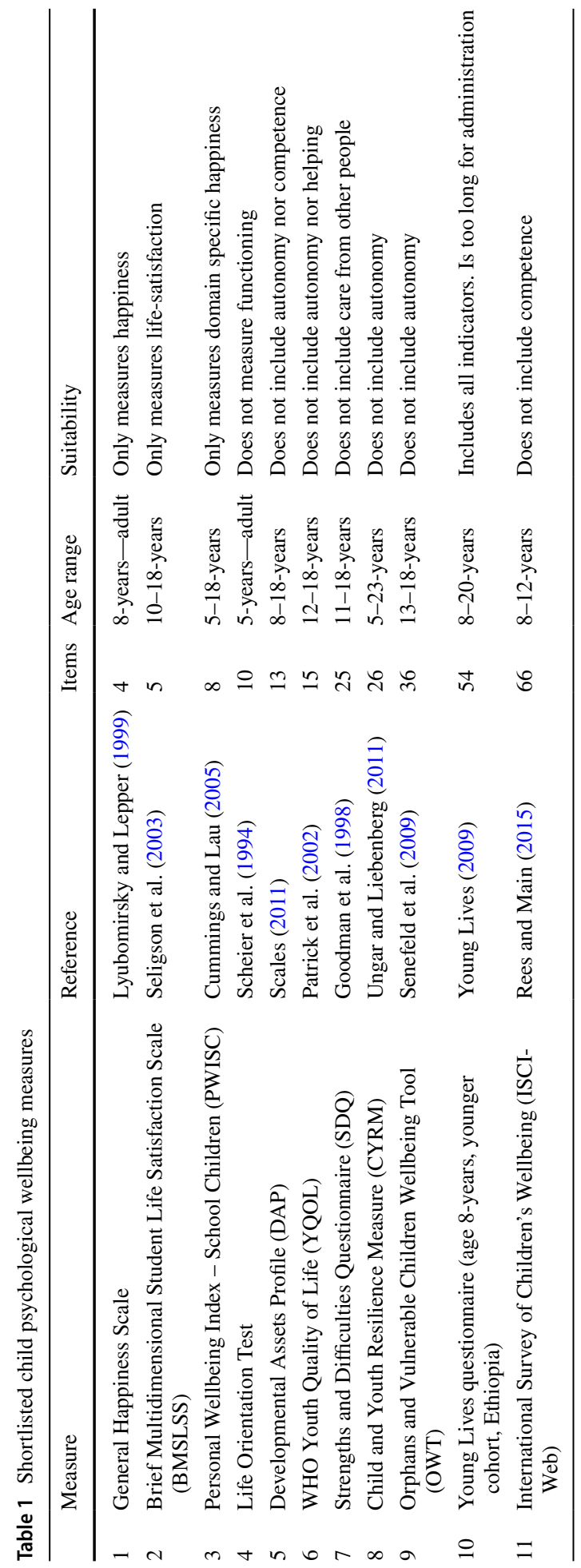




\subsection{Participants}

The qualitative investigation took place in two village communities that had agreed (with permission from the community leader) to participate in the evaluation pilot study. Children, adolescents, and adults were invited to take part in focus group interviews and the details of the research were explained to them verbally by a mother tongue Temne speaker. Participants gave verbal assent to participate. A total of 40 people participated. Recruited children and adolescents were aged 8-16-years. In Community A, there were four focus groups: group $1=6$ male children, group $2=6$ female children, group $3=6$ adult females, and group $4=7$ adult males $(\mathrm{N}=25)$. In Community $\mathrm{B}$ there were two mixed gender groups: group $1=7$ children (genders unrecorded by the fieldworkers), and group $2=4$ adult males and 4 adult females $(\mathrm{N}=15)$. No information on participants' social backgrounds was recorded.

\subsection{Procedures}

Semi-structured interviews were conducted in Temne by a members of a local research agency working alongside a member of the university research team. The interviews were audio recorded and transcribed directly into English by the mother tongue interviewer and checked for accuracy by the local research agency before being passed to the university research team for analysis. The interview schedule was in two parts.

\subsubsection{Indigenous Perceptions of Wellbeing Interview Schedule}

The first part of the interview schedule was adapted from the Wellbeing Exercise (Crivello et al., 2009). Participants were asked to think of a boy they knew aged between 5-years and 10 -years (the age range of our broader study) who was 'doing well', then state the reasons that the boy was doing well. They were asked to do the same for a girl they knew. Both child and adult participants responded to the same interview questions.

\subsubsection{Eudaimonic and Hedonic Wellbeing Interview Schedule}

The second part of the interview schedule was designed to test participants' understanding of the eight indicators in our framework of hedonic and eudaimonic wellbeing (Fig. 1), and to investigate whether the concepts related to participants' lived experiences of wellbeing in their communities. First, the interviewers explained the eight indicators in Western terms. The following explanation was transcribed and back translated from Temne: "Acceptance: both you and other people like the person that you are. Competence: you can do the things that you want to do very well. Safety: do you feel safe, are you afraid for your health or that you would be harmed. Autonomy: you can choose the things that you want to do for yourself. Care: other people look after you and are concerned for your safety and happiness. Reciprocity: you are a useful help to other people. Happiness: you feel happy. Problem solving: you can find a way to deal with or manage your problems". Second, the interviewer prompted discussion on each concept in the local context: "Do you think that acceptance is important for the wellbeing of children in this community... Do you think that competence is important for the wellbeing of children in this community, etc."? Respondents answered freely to the second set of questions on how each indicator might manifest in lived experiences in the community. 


\subsection{Analysis}

Codes and themes were generated using QDA Miner software. Then, coded text segments were imported into Microsoft Excel for sorting and a matrix analysis. The first author generated the initial coding schemes. These were checked for reliability by the seventh author. The analysis procedure for each section of the interview schedule is below.

\subsubsection{Indigenous Perceptions of Wellbeing Analysis}

These data were analyzed using inductive thematic analysis following Clarke et al. (2015). The first author read the transcripts for familiarity, generated initial codes by line by line reading of the transcripts, organized the initial codes into themes, reviewed the subthemes against the codes and the original transcripts to refine the coding system, and defined and named the final set of themes and codes. A total of 242 statements were coded.

\subsubsection{Eudaimonic and Hedonic Wellbeing Analysis}

The first author coded the data into the deductive hedonic and eudaimonic measure indicators (e.g., acceptance, autonomy). Then, they used the same inductive thematic analysis method outlined above (Clarke et al., 2015) to code all the data again, to create an inductive coding scheme that cut across the eight indicators. A total of 164 statements were coded into this deductive/inductive framework of hedonic/eudaimonic wellbeing.

\subsubsection{Interrater Coder Reliability}

We checked the robustness of the two inductive coding schemes (indigenous perceptions, and inductive hedonic/eudaimonic wellbeing) using a form of interrater coder reliability. The statements coded by the first author were extracted and randomly ordered using Microsoft Excel. The seventh author coded the first 25 randomly ordered statements from each coding scheme without knowing where they had been coded previously. The results were checked by the first and seventh authors. For the indigenous perceptions coding scheme, the inter-rater coder reliability was $96 \%$ (24 out of 25 statements coded into the same categories by the first and seventh author). For the inductive hedonic/eudaimonic coding scheme, the inter-rater coder reliability was $72 \%$ (18 out of 25 statements agreed on). The inter-rater coder difference was caused by 7 statements that could arguably be coded into multiple indicators of hedonia/eudaimonia.

\subsection{Results}

\subsubsection{Indigenous Perceptions of Wellbeing Qualitative Results}

Four main themes emerged for why children were perceived as doing well: being cared for by others, participating in the community, being educated, and physical wellbeing. Instances of communities, parents and teachers caring for the child were mentioned by male children and mixed gender adults (Table 2). Being cared for by parents and teachers was mentioned by male children and mixed gender children. Every focus group mentioned chores and being respectful and obedient to adults as an important part of community 
Table 2 Indigenous perceptions of wellbeing by age and gender

\begin{tabular}{|c|c|c|c|c|c|c|c|c|}
\hline Themes \& Codes & Sample Quote & $\mathrm{MC}$ & $\mathrm{FC}$ & $\mathrm{XC}$ & MA & FA & XA & Total \\
\hline \multicolumn{9}{|l|}{ Cared for by others } \\
\hline Community care & The people of the community love her (XA) & 1 & & & & & 1 & 2 \\
\hline Parental care & The parents really love the child (MC) & 6 & & & & & & 6 \\
\hline Teacher care & The teachers like him $(\mathrm{XC})$ & & & 2 & & & & 2 \\
\hline \multicolumn{9}{|c|}{ Community participation } \\
\hline Chores & He sweeps in the morning (FA) & 3 & 1 & 1 & 3 & 9 & 11 & 28 \\
\hline Friendly greets & She greets in the morning (MA) & & 1 & 1 & 4 & 2 & 4 & 12 \\
\hline Helpful & She helps the people in the community (FC) & & 5 & & & & 5 & 10 \\
\hline Love parents & She likes her parents (MA) & & & & 3 & & 1 & 4 \\
\hline Not aggressive & She is not using abusive language (FC) & 1 & 3 & & & 2 & 1 & 7 \\
\hline Religiosity & He prays $(\mathrm{MC})$ & 2 & & & & & & 2 \\
\hline Respect obedience & He is obedient in the community (MC) & 5 & 14 & 2 & 7 & 13 & 14 & 55 \\
\hline Work & He is supporting me to do my work (FA) & & 1 & & & 1 & & 2 \\
\hline \multicolumn{9}{|l|}{ Education } \\
\hline Brilliant & The child is brilliant in school (MA) & 1 & 4 & 4 & 3 & 1 & 4 & 17 \\
\hline School attendance & She is regular in school (XA) & 3 & 1 & 2 & 3 & 4 & 7 & 20 \\
\hline School happiness & She likes the school (MA) & & 1 & & 2 & & & 3 \\
\hline Studying & He likes studying (FC) & 1 & 5 & 2 & 3 & 2 & 2 & 15 \\
\hline \multicolumn{9}{|l|}{ Personal wellbeing } \\
\hline Eating & He eats good food (XC) & 3 & & 2 & 3 & 2 & 1 & 11 \\
\hline Grooming & The child is clean (FC) & 3 & 11 & & & 1 & 3 & 18 \\
\hline Healthy & She is healthy (MA) & & & 2 & 6 & & 7 & 15 \\
\hline Play & The boy I chose has time to play (MC) & 1 & & & 2 & & & 3 \\
\hline Sleeping & The child always has enough rest (MA) & 4 & & & 2 & & & 6 \\
\hline Total & & 34 & 47 & 18 & 41 & 37 & 61 & 238 \\
\hline
\end{tabular}

$F$ female, $M$ male, $X$ mixed gender group, $C$ child, $A$ adult

participation, with other less commonly mentioned aspects of being helpful, greeting others, loving parents, not being aggressive, being religious, and working in the community. Every focus group also mentioned children who were 'brilliant' (a Sierra Leonian term for holistic competence) at school, and who regularly attended school and studied effectively. Finally, all focus groups except the female children discussed having enough to eat as an indicator of child wellbeing, and other less common aspects of grooming (dress, clean, braid hair), sleeping, playing, and being healthy as indicators of wellbeing. An overview of these results is presented in Table 2 .

\subsubsection{Eudaimonic and Hedonic Wellbeing Qualitative Results}

Using a matrix analysis (Table 3), we uncovered that each of the eight measurement indicators had ecological validity in multiple aspects of the participants' lives (e.g., the deductive theme of care was mentioned in relation to the inductive themes of being cared for by others, community participation, education, psychological wellbeing, and physical wellbeing). 
Table 3 displays the overlaps between the two sets of themes and gives sample quotes for the most common overlaps.

The qualitative investigation enabled us to elicit participants' perspectives on child wellbeing, by using an exploratory approach and by questioning participants about the eight indicators of hedonic/eudaimonic wellbeing used in our measure. We briefly conclude on the key findings that are most relevant to our measure development study.

First, we found that parallel themes regarding child wellbeing emerged in both the inductive coding frames. In both discussions (about why a child was doing well, and about the eight indicators), participants mentioned the importance of caring for children, children contributing to the community, and children's physical wellbeing. However, participants only elaborated on issues of psychological wellbeing and safety when discussing the eight indicators. Second, participants' responses about why a child was doing well, were far simpler than those generated in the discussion about the eight indicators. Possibly, rapport was built with the interviewers during the first discussion, therefore participants felt freer to speak in detail during the second discussion. Another possibility is that the eight indicators discussion provoked more detailed responses, as participants were able to connect these concepts back to the simpler, more concrete points they had discussed earlier (e.g., doing chores, attending school). Their responses detailed children feeling happy and good about things, adults helping children develop, and concerns about children's safety from physical, social, and supernatural dangers. More qualitative research is needed to explore why this more tacit system of childhood functioning and wellbeing (e.g., van Manen \& Levering, 1996) only emerged when prompted in discussion about the eight indicators. Third, and most importantly, all the measurement indicators were easily understood by participants who were able to give ecologically valid examples of these occurring in their local contexts (Table 3). We expand on this issue in the overarching discussion.

\section{Study 3: Development of the Wellbeing Measure}

We designed and tested a measure of children's hedonic and eudaimonic wellbeing for use in rural Sierra Leone, that was informed by our theoretical perspective and the qualitive investigation of participant's perceptions of children's wellbeing. We developed the wellbeing measure during a longitudinal pilot study (October 2017 and May 2018) of the intervention administration and evaluation, that spanned the 2017-2018 school year. The pilot study gave us an ideal opportunity to develop the wellbeing measure across two time points, with the second time point informed by the qualitative focus group results (Study 2).

\subsection{Participants}

The pilot study (Sect. 5) and main study (Sect. 6) methodology was approved by the university's Human Research Ethics Committee. As described in the research context section, the quantitative study was part of a mixed methods evaluation of an intervention for promoting literacy and wellbeing in rural Sierra Leone. The research funder is a non-governmental organization (NGO) with nearly 25-years' experience of providing humanitarian aid in Sierra Leone. The NGO identified provided the research team with a list of the 118 communities and 137 primary schools in the district where the intervention took place. 


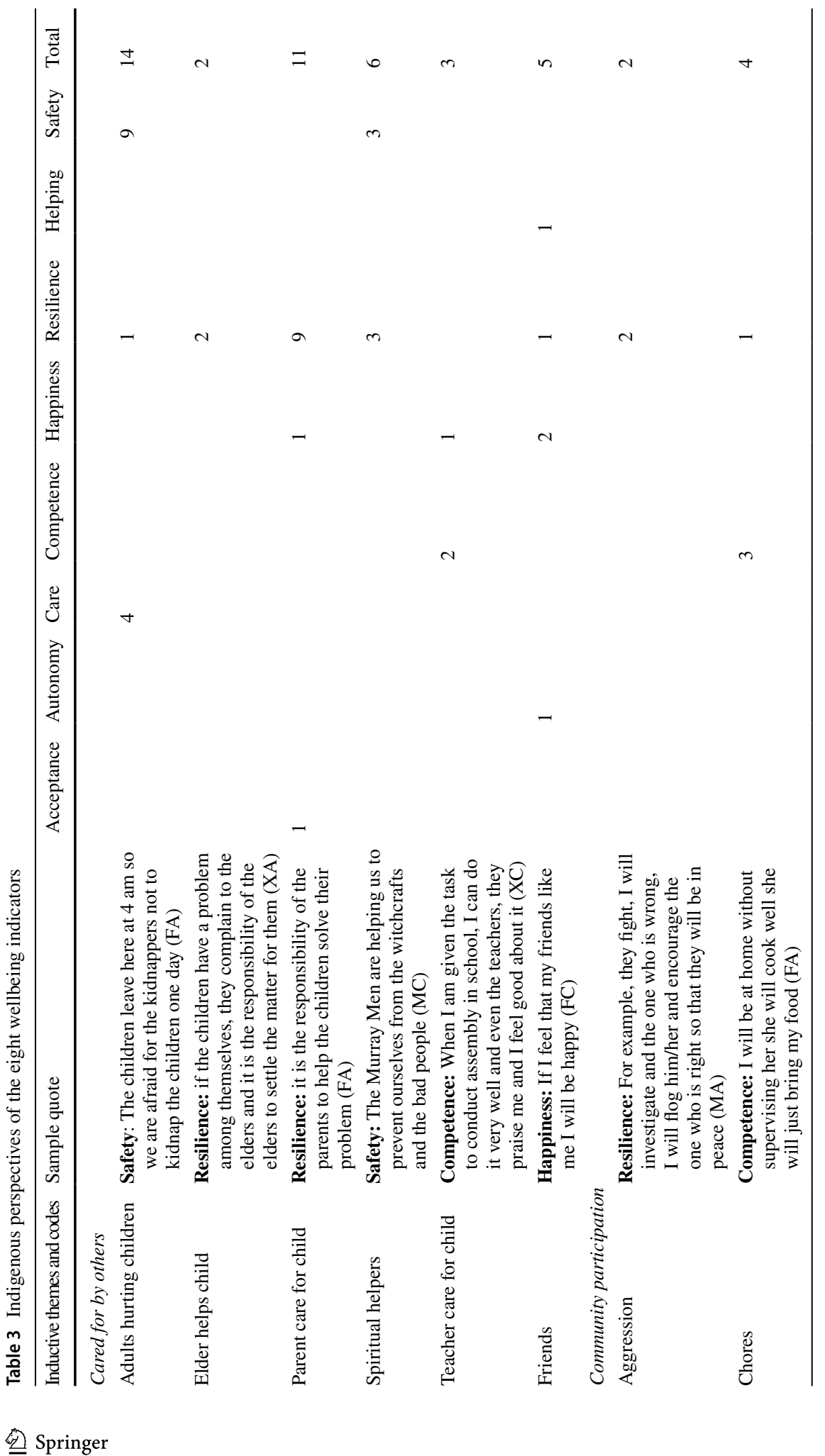




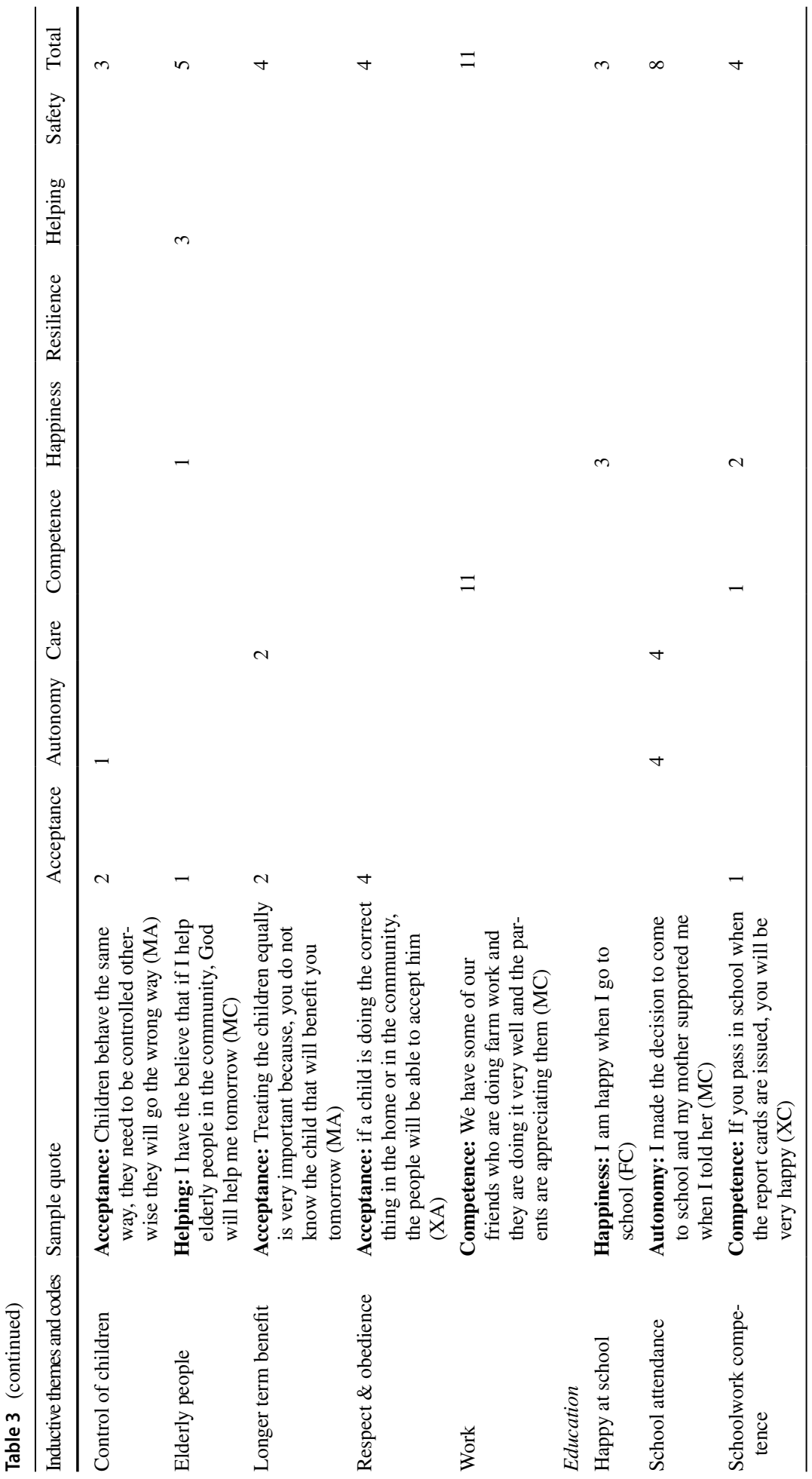




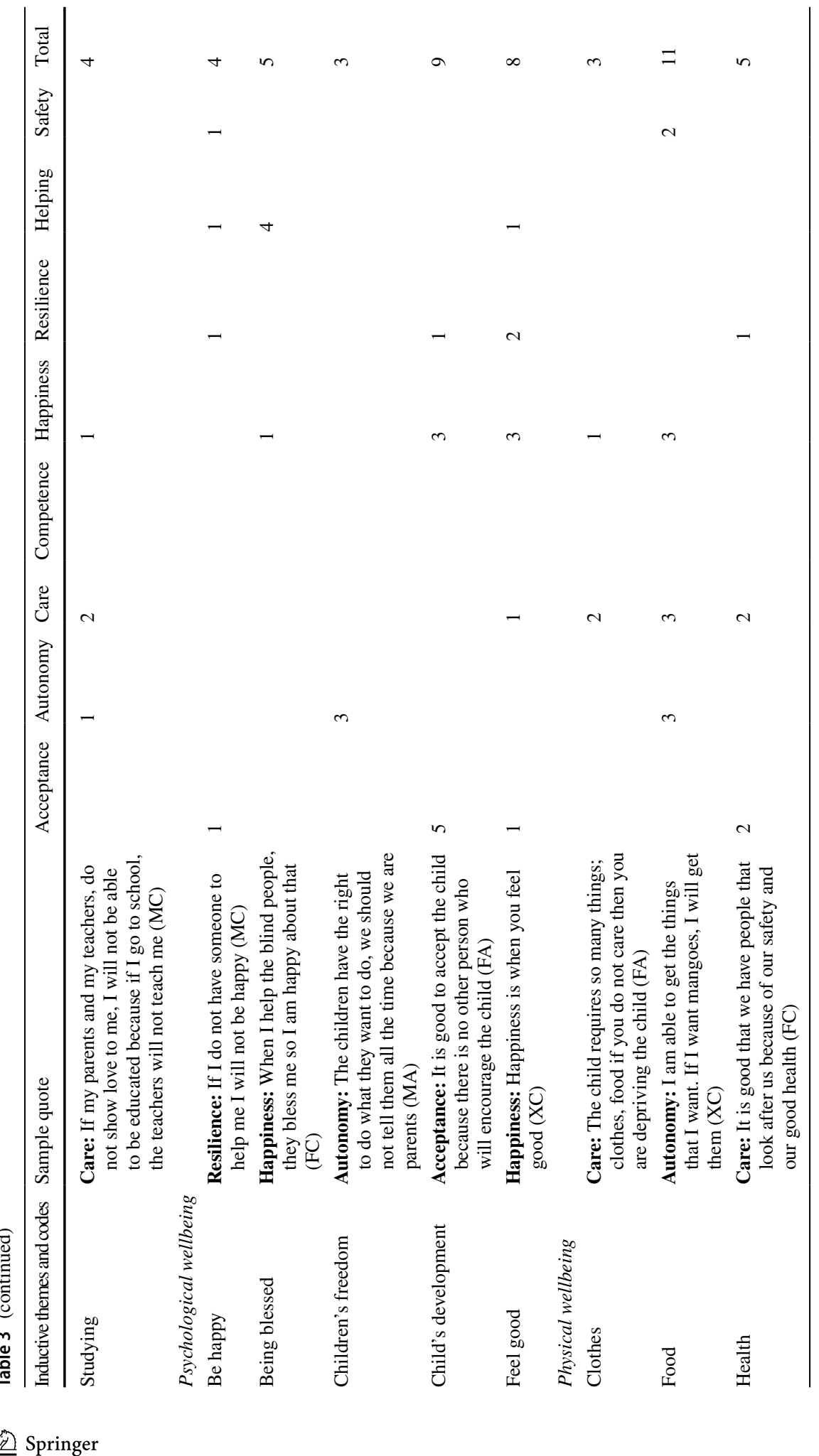




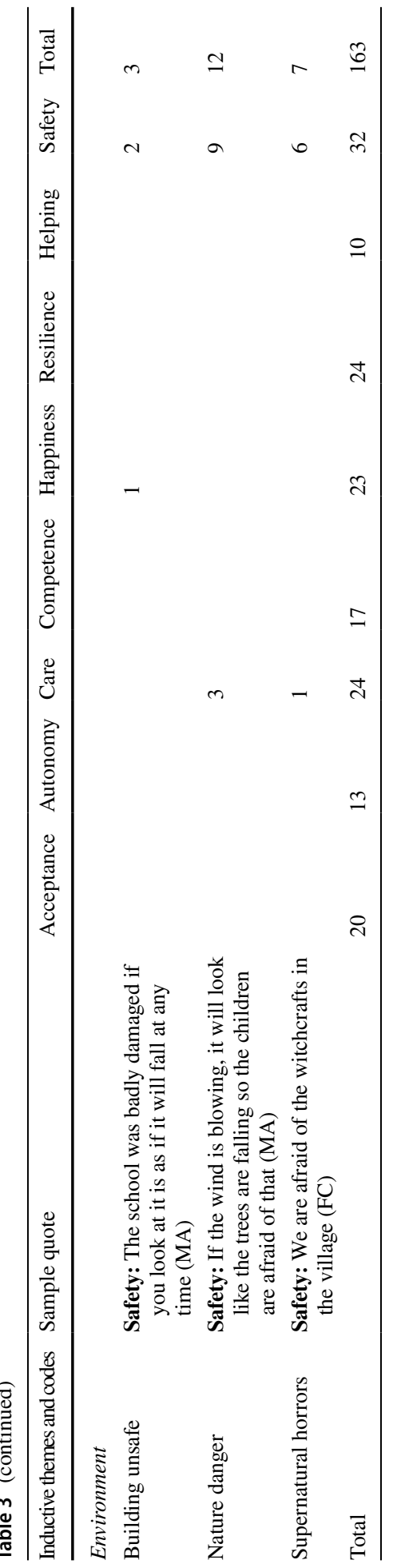


Only schools with Classes 1-6 (equivalent Grades 1-6), and more than 15 children in Class 1 , were eligible to participate. This resulted in 105 eligible schools.

Next, the NGO invited each community leader to participate in the research. Once this was agreed, the NGO met with each school headteacher and class teachers to provide them with written and verbal information about the research. Headteachers signed a memorandum of understanding to participate. Information sessions with teachers, and parents of Class 1 children, were held in each school, staffed by the NGO and the research team. Attending parents signed the class register and were given oral information about the study in mother tongue, with written information in English and an opt-out consent form. Only children of attending parents who did not return the opt-out consent form were eligible to participate. Children's verbal assent to participate was recorded by fieldworkers before each interview.

Up to 30 children per class were selected to participate by the researchers, who used random sampling to select the children if the class had more than 30 children in total. After the recruitment process, 95 communities agreed to participate, and across those communities, 99 schools (one large community had 4 schools, the remainder had 1 each) consented to participation. Ten schools drawn from one chiefdom were selected for the evaluation pilot study which was conducted in October 2017 and May 2018. The remaining 92 schools were involved in the main study, where data collection began in May 2019.

The pilot study sample $(\mathrm{N}=200)$ was $50 \%$ female; and had an average age of 6.92 years $(\mathrm{SD}=1.56$, age range: 3 -years to 10 -years). This wide age range was due to the presence of older children/early adolescents in the first year of school who were repeating the year or joining the school for the first time; and to the presence of younger children who were placed in Class 1 for repeated years until they were old enough to move to Class 2 (the practice is often a 'Class 1 a' with younger children and a 'Class 1 b' with older children). At home, $95 \%$ of the sample spoke Temne and 5\% spoke Krio. Regarding religion, 64\% were self-reported Muslim and 36\% were self-reported Christian.

\subsection{Procedures}

Before each data collection timepoint, fieldworkers from a Sierra Leonean research agency were trained for 3-days in Freetown. Each school visit was carried out across one to three days, by a team of four fieldworkers and one fieldwork supervisor. Children were interviewed individually in a sheltered place in the school yard. Data were entered into handheld tablet computers and were uploaded to the fieldwork agency's secure database at the earliest opportunity. The wellbeing measure was administered on the tablets using Survey CTO.

Fieldworkers followed a written assessment protocol for administering the wellbeing measure. Fieldworkers pointed to each cup on the 5-point scale and explained what it stood for. The following is an example from the protocol: [POINT TO THE FIRST GLASS]: This glass is empty: It means NEVER - that something "never" happens. After fieldworkers had reviewed each cup and its meaning, they asked the child to repeat the meaning of each picture. After the child had demonstrated understanding, the fieldworkers read out each item and asked the child to point to the picture that best described how often this item occurred in their lives. Items were read in order of each domain (personal, peer, teachers, family) to help children visualize and remember their experiences with specific groups of people. 


\subsection{Wellbeing Measure}

The pilot study allowed us to develop the Child and Adolescent Personal and Social Assessment of Wellbeing (CAPSAW). This is a 32-item measure of wellbeing across four dimensions: a global dimension, and dimensions of experience with peers, teachers, and family. Each dimension comprises eight items each capturing different indicators of eudaimonia (autonomy, competence, resilience (problem solving), and helping others) and hedonia (being cared for, safety, happiness, and acceptance). The items are measured on a fivepoint Likert type scale $(1=$ never, $2=$ rarely, $3=$ sometimes, $4=$ usually, $5=$ always $)$. More details on the measure can be found in the indicator, dimension, and scale development sections. The first eight items are displayed in Table 4.

We developed the wellbeing measure during a longitudinal pilot study (October 2017 and May 2018) of the intervention administration and evaluation, that spanned the 2017-2018 school year. The pilot study gave us an ideal opportunity to develop the wellbeing measure across two time points, with the second time point informed by the qualitative focus group results (Study 2).

Table 4 Personal psychological wellbeing subscale

\begin{tabular}{lll}
\hline & & Personal \\
\hline Hedonia & Acceptance & 1. Do you feel good about who you are? (H) \\
& Happiness & 2. Are you happy in general? (H) \\
Care & 3. Do you think people care about you? (H) \\
& Safety & 4. Do you feel safe in general? (H) \\
Eudaimonia & Autonomy & 5. Can you do the things you want to do in your life? (E) \\
& Competence & 6. Can you do things well for yourself? (E) \\
& Resilience & 7. If you have a problem, can you find a way to deal with it? (E) \\
& Helping & 8. Do you think you are helpful to other people? (E) \\
\hline
\end{tabular}

$H$ hedonia item, $E$ eudaimonia item

\subsubsection{Indicator Development}

For the first wave of the pilot study (October 2017), we developed an initial set of nine indicators of wellbeing. Eight of these were adapted from the measures identified in our systematic review (Study 1) and included an indicator of children's physical wellbeing (whether children felt healthy). Acknowledging the variety of definitions of resilience existing in the literature, we selected problem solving as a core method of overcoming challenges. The ninth indicator was constructed by the research team to measure violence prevention which was central to the NGO's intervention (do other people beat you).

After collecting and analyzing the October 2017 data, we decided to remove the violence and health indicators. In line with our ethnographic observations of violence in the communities, we also found that there was little quantitative value in measuring how much children were beaten by others as an indicator of wellbeing, because being beaten was a commonplace occurrence in school and family contexts and there was very little statistical variation in this indicator. Furthermore, we decided to remove the health item to keep the measure focused on psychological indicators of wellbeing. So as not to lose information on 
child subjective health from the main study, we designed a new set of culturally appropriate items about nutrition, hydration, hygiene, and physical illness to create a subsidiary measure of physical wellbeing that we do not report on here.

As well as removing the health and violence indicators from the wellbeing measure, we added a new indicator of children helping others, informed by our qualitative focus groups and by consultation with an ethnographer on the research team who was conducting an indepth case study of two village communities. The concept of helping others is core to relational wellbeing (e.g., White, 2015) and to our social evolutionary perspective on hypercooperative societies (Tomasello \& Vaish, 2013).

In the second wave of the pilot study (May 2018), we administered the revised measure covering the final eight indicators of eudaimonia (autonomy, competence, resilience, and helping others) and hedonia (being cared for, happiness, safety, and acceptance), designed to cover global eudaimonia and hedonia, and domain specific eudaimonia and hedonia (peers, teachers, and family). We describe the development of these four dimensions next.

\subsubsection{Dimension Development}

We measured each of the eight indicators within a global dimension (the child's perception of their overarching eudaimonia and hedonia), and within domain specific dimensions of the child's perception of eudaimonia and hedonia in relation to peer, teacher, and family developmental contexts. To do this, we first constructed the items for the global dimension. Then, we reworded each global dimension item (e.g., do you feel happy in general?) into a parallel item for each domain specific dimension (e.g., do your teachers make you feel happy?). The repetition of each of the eight indicators across the four dimensions created the final 32-item Child and Adolescent Personal and Social Assessment of Wellbeing (CAPSAW).

\subsubsection{Scale Development}

Studies of children in Australia and the United States have found that participants as young as age five are able to assess whether items are true of them on temporal scales (Marsh et al., 1998) ranging from always to never (Varni et al., 2007). Following these studies, we developed a 5-point scale for assessing the 32 items with the scale points of: never, rarely, sometimes, most of the time, and always. In scale pre-testing we discovered that children in rural Sierra Leone did not understand the 'thumbs up' signal and tended to indicate that everything was good. We therefore developed a 'neutral' image of drinking cups with different levels of water, to represent the scale points of always to never (Fig. 2).
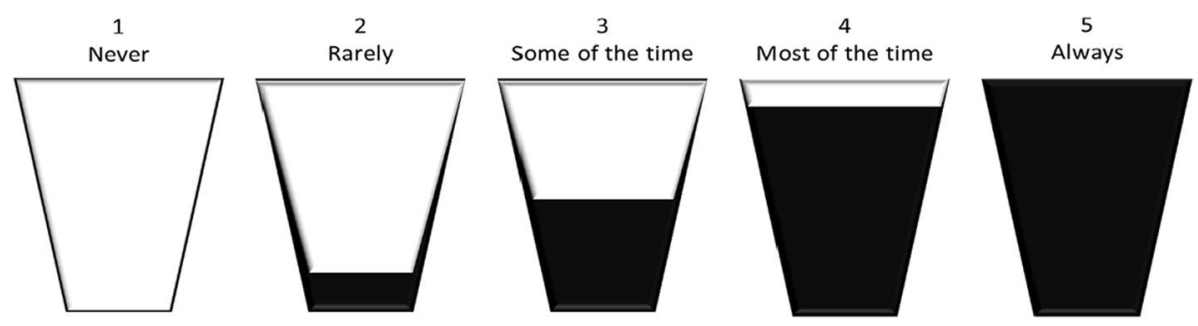

Fig. 2 Visual prompt for the CAPSAW scale 


\subsubsection{Translation and Cultural Adaptation}

Before administering the measure with children, we reviewed each aspect of the measure (item wording, scale wording, and visual prompt) with a team of mother tongue experts in Freetown. Reflecting the languages of our pilot sites, the team consisted of one native Temne speaker, and two native Krio speakers. First, the group discussed the meaning of each item with the researchers to establish whether there was a parallel in the local culture. Although every item was recognized conceptually, our discussion led to subtle changes in the way the concepts were expressed, for example the original item I am happy with the kind of person I am was changed to Do you feel good about who you are? Next, each item was translated into Temne, then into Krio, then back translated into English from Temne and Krio, to ensure comparability across the three languages.

\subsection{Analysis Plan}

We used the second wave of pilot study data to test the measure's dimensional validity with a focus on whether the eight indicators within each dimension (global, peer, teacher, family) fit the anticipated structure of hedonia and eudaimonia. First, we generated descriptive statistics and reliability analyses for all measured dimensions using SPSS version 24. Second, we computed an exploratory factor analysis (EFA) of the measure in Mplus version 8.0, including schools as a clustering variable to produce adjusted standard errors. We used the Mplus default rotation method for EFA, Geomin rotation (Yates, 1987), which assumes that all items are correlated. We constrained the EFAs to extracting two factors, to provide a strict test of our assumption that the items would load differentially onto factors representing hedonia and eudaimonia. EFA models were assessed for model fit using the root mean square error of approximation (RMSEA) (Steiger, 1990) and comparative fit index (SFI) (Bentler, 1990). We used cut off points to assess whether the fit between the models and the data was good: $\mathrm{CFI}<0.9$ and RMSEA $<0.5$ (Hu \& Bentler, 1999).

\subsection{Results}

Descriptive statistics (mean values, standard deviations, and correlations) are presented for the pilot study in Table 5 .

\subsubsection{Reliability Analysis}

We computed Cronbach's alphas to examine whether each dimension could be analyzed as two factors: hedonia (autonomy, competence, resilience, and helping others) and hedonia (being cared for, safety, happiness, and acceptance). The hedonic and eudaimonic factors were internally reliable for the personal dimension $(\alpha=0.72 \& \alpha=0.74)$ and the peer dimension $(\alpha=0.70 \& \alpha=0.70)$. Hedonia was acceptably reliable for the teacher $(\alpha=0.77)$ and the family $(\alpha=0.76)$ dimensions, but eudaimonia was not reliable for either dimension (teacher $\alpha=0.64$, family $\alpha=0.62$ ). 
Table 5 Pilot study descriptive statistics

\begin{tabular}{lllllllll}
\hline & 1 & 2 & 3 & 4 & 5 & 6 & 7 & 8 \\
\hline $\mathrm{N}$ & 200 & 200 & 200 & 200 & 200 & 200 & 200 & 200 \\
Min & 2 & 1.25 & 1.75 & 1.75 & 1.5 & 1.75 & 2.25 & 2.25 \\
Max & 5 & 5 & 5 & 5 & 5 & 5 & 5 & 5 \\
M & 3.86 & 3.05 & 3.55 & 3.13 & 3.64 & 3.11 & 4.31 & 3.62 \\
SD & 0.68 & 0.86 & 0.61 & 0.64 & 0.62 & 0.59 & 0.65 & 0.67 \\
$\alpha$ & 0.72 & 0.74 & 0.70 & 0.70 & 0.77 & 0.60 & 0.76 & 0.62 \\
1. Hedonia & 1 & & & & & & & \\
2. Eudaimonia & $.460 * * *$ & 1 & & & & & & \\
3. Peer hedonia & $.554 * * *$ & $.555^{* * *}$ & 1 & & & & & \\
4. Peer eudaimonia & $.453 * * *$ & $.665 * * *$ & $.703 * * *$ & 1 & & & \\
5. Teacher hedonia & $.524 * * *$ & $.316^{* * *}$ & $.533 * * *$ & $.394 * * *$ & 1 & & \\
6. Teacher eudaimonia & $.447 * * *$ & $.620 * * *$ & $.601 * * *$ & $.667 * * *$ & $.540 * * *$ & 1 & & \\
7. Family hedonia & $.622 * * *$ & $.268 * * *$ & $.555^{* * *}$ & $.365 * * *$ & $.643 * * *$ & $.390 * * *$ & 1 \\
8. Family eudaimonia & $.557 * * *$ & $.639 * * *$ & $.679 * * *$ & $.658 * * *$ & $.470 * * *$ & $.698 * * *$ & $.548 * * *$ & 1 \\
\hline
\end{tabular}

\subsubsection{Exploratory Factor Analysis}

Separate EFA models were run for the personal, teacher, family, and peer dimensions. We made one minor adjustment to the personal model by specifying the association between the $3^{\text {rd }}$ and $8^{\text {th }}$ items to bring the RMSEA down from 0.9 to 0.5 . The original and adjusted models were compared to ensure that the factor structure was unaltered. The models fit the data well (Table 7).

The item factor loadings for the personal, peer, family, and teacher dimensions are displayed in Table 8. The highest loading of an individual item is emphasized using bold font, and where individual item loadings are equivalent to 0.01 , both loadings are emboldened. The results presented an overarching pattern of hedonia and eudaimonia as anticipated, with some variation across dimensions. The results of the EFA showed that 15 out of 16 hedonia items loaded onto the first factor (94\% accuracy), and 14 out of 16 eudaimonia items loaded onto the second factor ( $88 \%$ accuracy). The anticipated structure was most reliable for the personal subscale (with all items loading as expected except for the safety item which loaded equally onto both factors), and for the teacher subscale (with all items loading as expected). In the family subscale all the hedonia items loaded onto the first factor, and all eudaimonia items loaded onto the second factor except for the problem-solving item. Finally, all the peer items loaded as expected, except for the safety and competence items which loaded onto the alternative factors.

\section{Study 4: Extended Validity Testing}

We further examined the properties of the wellbeing measure using data from the first wave of the main intervention evaluation. This allowed us to test the dimensional validity in a much larger sample, and to perform additional tests of the measure's concurrent and predictive validity. 


\subsection{Participants}

Participants were drawn from the 92 schools recruited into the study that did not take part in the pilot study. For full details of participant recruitment please see Sect. 5.1 above. The main study sample $(N=2382)$ was $51 \%$ female; and had an average age of 7.81 years ( $S D=1.67$, age range 4-years to 13 -years). At home $59 \%$ of the sample spoke Temne, $28 \%$ spoke Krio, 9\% spoke Kuranko, 3\% spoke Limba and 1\% spoke English or Mende.

\subsection{Procedures}

The same procedures reported for the pilot study were used in the main study.

\subsection{Measures}

\subsubsection{Eudaimonic and Hedonic Wellbeing}

We used the same version of the CAPSAW reported on in the pilot study to measure children's eudaimonic and hedonic wellbeing. However, because the full measure took approximately 15-min to administer, we only measured the personal and teacher dimensions (16 items in total) in the main study in May 2019 to facilitate an additional literacy measure which we do not report on here, a fuller set of demographic items, and the measure of motivation/persistence which we describe below. Using only half of the wellbeing measure enabled a shorter administration time which was helpful for protecting the children who were interviewed outdoors in the school yards during the daytime heat.

\subsubsection{Motivation/Persistence}

In the main study, we used selected items from the 42-item Multidimensional School Engagement Scale (Wang et al., 2017) to measure children's school perceptions. After an exploratory factor analysis in SPSS (not reported here) we retained four items that measured motivation/persistence: Are you interested in what you are learning at school?, Do you always try your best in school?, Do you keep trying even when you get stuck on your schoolwork?, and Do you work hard when things are difficult at school? Each item was measured on a 5 -point scale $(1=$ never, $2=$ rarely, $3=$ sometimes, $4=$ usually, $5=$ always $)$. The motivation variable $(\alpha=0.84)$ was used to test the wellbeing measure's concurrent validity.

\subsection{Analysis Plan}

We used the same method of testing dimensional validity as described in the pilot study. First, we computed Cronbach's alphas for the two dimensions in SPSS version 24. Second, we conducted an exploratory factor analysis in Mplus version 8.0 using schools as the clustering variable, Geomin rotation, and extracting two factors to check for the presence of hedonia and eudaimonia.

Third, we checked for concurrent validity using the motivation measure. We assumed that motivation would associate with hedonia and eudaimonia, because motivation is conceptualized as a core component of wellbeing in other perspectives (e.g., Deci \& Ryan, 
2008; McLellan \& Steward, 2015). Concurrent validity was tested using Pearson's twotailed correlation.

Finally, we used path modelling in MPlus 8.0 to examine the predictive validity of the personal and teacher hedonia and eudaimonia sub-scales, with child gender, child age, and child home language as predictors. These predictors of wellbeing were selected based on the results of in-depth, intergenerational qualitative case studies of four rural Sierra Leonian village communities (Devine et al., in press a, b). In these case studies, children's age was central to child and adult expectations of child obedience and punishment; and impacted children's levels of responsibility in the community with older children contributing more to economic activities and younger children contributing mainly to domestic activities. In turn, these activities were cited as important for children's wellbeing (Devine et al., in press a), e.g., "We have some of our friends who are doing farm work and they are doing it very well and the parents are appreciating them" (male child, Table 3). Gender also impacted how opportunities for wellbeing were structured in the case study communities, with female roles centering on domestic forms of caring for families, and male roles centering on providing economic resources to families and physical protection (Devine et al., in press b). Finally, language was included as a proxy for the different cultural groups that were predominant in the sample. The languages of Krio, Temne and Kuranko were entered as binary variables $(1=$ speaks language at home, $0=$ does not speak language at home).

\subsection{Results}

Descriptive statistics (mean values, standard deviations and correlations) are presented in Table 6.

\subsubsection{Reliability Analysis}

The hedonic and eudaimonic components of the dimensions were internally reliable for the personal dimension $(\alpha=0.71 \& \alpha=0.75)$. Hedonia was reliable for the teacher dimension $(\alpha=0.84)$ but eudaimonia was not $(\alpha=0.60)$.

\subsubsection{Exploratory Factor Analysis}

Separate EFA models were run for the personal and teacher dimensions and fit the data well (Table 7). The 16 item factor loadings for the personal and teacher dimensions (8-items each) are displayed in Table 8. Across the 16 items, 14 loaded as expected (88\% accuracy). Within the personal subscale, $100 \%$ of items loaded as anticipated. The unanticipated loadings occurred in the teacher subscale, where the competence and problem-solving items loaded equivalently ( 0.05 difference) onto both factors. However, a clear teacher hedonia component was apparent, with the hedonia items loading onto the first factor much more strongly $(0.78-0.71)$ than the two eudaimonia items (both 0.49$)$.

\subsubsection{Concurrent Validity}

Using the motivation measure, we tested the concurrent validity of the personal and teacher dimensions. The motivation measure was significantly associated with personal hedonia $(r=0.511, p<0.001)$ and eudaimonia $(r=0.406, p<0.001)$ and teacher hedonia $(r=0.504, p<0.001)$ and eudaimonia $(r=0.588, p<0.001)$. This demonstrates that the 
Table 6 Main study descriptive statistics

\begin{tabular}{|c|c|c|c|c|c|}
\hline & 1 & 2 & 3 & 4 & 5 \\
\hline $\mathrm{N}$ & 2382 & 2382 & 2382 & 2382 & 2382 \\
\hline Min & 2.5 & 1 & 1.25 & 1.25 & 1.25 \\
\hline Max & 5 & 5 & 5 & 5 & 5 \\
\hline M & 4.34 & 3.26 & 4.27 & 3.49 & 4.02 \\
\hline SD & 0.53 & 0.84 & 0.63 & 0.69 & 0.70 \\
\hline$\alpha$ & 0.71 & 0.75 & 0.82 & 0.64 & 0.84 \\
\hline 1. Hedonia & 1 & & & & \\
\hline 2. Eudaimonia & $.291 * * *$ & 1 & & & \\
\hline 3. Teacher hedonia & $.633^{* * *}$ & $.237 * * *$ & 1 & & \\
\hline 4. Teacher eudaimonia & $.424 * * *$ & $.658 * * *$ & $.490 * * *$ & 1 & \\
\hline 5. Motivation/persistence & $.511^{* * *}$ & $.406 * * *$ & $.504 * * *$ & $.588 * * *$ & 1 \\
\hline
\end{tabular}

Table 7 Model fit statistics for the EFA models

\begin{tabular}{lllllllll}
\hline & \multicolumn{3}{l}{ Pilot study } & & & & \multicolumn{2}{l}{ Main study } \\
\cline { 2 - 3 } \cline { 8 - 9 } \cline { 7 - 8 } & Personal & Peer & Teacher & Family & & Personal & Teacher \\
\hline RMSEA & 0.05 & 0.00 & 0.04 & 0.05 & & 0.05 & 0.05 \\
CFI & 0.98 & 1.00 & 0.99 & 0.99 & & 0.96 & 0.97 \\
\hline
\end{tabular}

wellbeing measure dimensions were positively associated with the related construct of school motivation.

\subsubsection{Predictive Validity}

We computed a path model with personal and teacher hedonia and eudaimonia as the outcome variables, and gender, age, and home language as predictors. The model was an excellent fit to the data $(\mathrm{CFI}=1, \mathrm{RMSEA}=0)$. Being female predicted higher personal hedonia $(b=0.06, p=0.014)$, personal eudaimonia $(b=0.05, p=0.023)$, and teacher eudaimonia $(b=0.06, p=0.009)$. Being older predicted higher personal eudaimonia $(b=0.16$, $p<0.001)$ and teacher eudaimonia $(b=0.09, \mathrm{p}<0.001)$. Speaking Krio $(28 \%$ of participants, $n=673)$ predicted higher personal hedonia $(b=0.31, p<0.001)$ and eudaimonia $(b=0.13, p=0.051)$, and higher teacher hedonia $(b=0.25, p<0.001)$. Speaking Kuranko (9\% of participants, $n=216)$ predicted higher personal hedonia $(b=0.23, p<0.001)$ and teacher hedonia $(b=0.25, p<0.001)$ and eudaimonia $(b=0.12, p=0.022)$. Speaking Temne (59\% of participants, $n=1398$ ) did not associate with any outcome. These results demonstrate that the measured dimensions were sensitive to detecting cultural variation (gender and language), and age variation, in children's reports of their hedonic and eudaimonic wellbeing. 
Table 8 CAPSAW items and factor loadings

\begin{tabular}{|c|c|c|c|c|}
\hline \multirow[t]{2}{*}{ Items } & \multicolumn{2}{|c|}{ Pilot study } & \multicolumn{2}{|c|}{ Main study } \\
\hline & $\mathrm{F} 1$ & $\mathrm{~F} 2$ & F1 & $\mathrm{F} 2$ \\
\hline
\end{tabular}

Personal dimension

1. Do you feel good about who you are? (H)

$\begin{array}{llll}\mathbf{0 . 7 5} & 0.23 & \mathbf{0 . 6 2} & 0.15\end{array}$

2. Are you happy in general? $(\mathrm{H})$

$\begin{array}{llll}\mathbf{0 . 6 0} & 0.43 & \mathbf{0 . 7 8} & 0.31\end{array}$

3. Do you think people care about you? $(\mathrm{H})$

$\begin{array}{lllll}\mathbf{0 . 5 3} & 0.26 & \mathbf{0 . 3 4} & 0.14\end{array}$

4. Do you feel safe in general? (H)

$\begin{array}{llll}0.54 & 0.55 & 0.74 & 0.30\end{array}$

5. Can you do the things you want to do in your life? (E)

$\begin{array}{lllll}0.27 & \mathbf{0 . 8 4} & 0.29 & \mathbf{0 . 7 0}\end{array}$

6. Can you do things well for yourself? (E)

$\begin{array}{llll}0.32 & \mathbf{0 . 7 1} & 0.42 & \mathbf{0 . 6 7}\end{array}$

7. If you have a problem, can you find a way to deal with it? (E)

$\begin{array}{llll}0.06 & \mathbf{0 . 5 2} & 0.13 & \mathbf{0 . 5 2}\end{array}$

8. Do you think you are a help to other people? (E)

$\begin{array}{llll}0.32 & \mathbf{0 . 5 1} & 0.29 & \mathbf{0 . 7 6}\end{array}$

Teacher dimension

9. Do your teachers like you for who you are? $(\mathrm{H})$

$\begin{array}{llll}\mathbf{0 . 7 6} & 0.36 & \mathbf{0 . 7 0} & 0.26\end{array}$

10. Do your teachers make you feel happy? $(\mathrm{H})$

$\begin{array}{lllll}\mathbf{0 . 6 2} & 0.60 & \mathbf{0 . 7 8} & 0.18\end{array}$

11. Do you think your teachers care about you? $(\mathrm{H})$

$\begin{array}{llll}\mathbf{0 . 6 9} & 0.46 & \mathbf{0 . 7 5} & 0.25\end{array}$

12. Do you feel safe with your teachers? $(\mathrm{H})$

13. Do your teachers allow you to do the things you want to do? (E)

$\begin{array}{llll}\mathbf{0 . 6 2} & 0.37 & \mathbf{0 . 7 1} & 0.30\end{array}$

$\begin{array}{lllll}0.26 & \mathbf{0 . 3 2} & 0.30 & \mathbf{0 . 4 5}\end{array}$

14. Do your teachers think you do things well in school? (E)

$\begin{array}{llll}0.37 & 0.39 & 0.49 & 0.44\end{array}$

15. Do your teachers help you if you have a problem? (E)

$\begin{array}{llll}0.48 & 0.61 & 0.49 & 0.42\end{array}$

16. Do you think you are a help to your teachers? (E)

$\begin{array}{llll}0.45 & \mathbf{0 . 7 9} & 0.27 & \mathbf{0 . 7 9}\end{array}$

Family dimension

17. Do your family like you for who you are? (H)

0.680 .17

18. Do your family make you feel happy? (H)

0.66 0.58

19. Do you think your family care about you? $(\mathrm{H})$

0.61 0.43

20. Do you feel safe with your family? (H)

$\mathbf{0 . 7 2} 0.35$

21. Do your family allow you to do the things you want to do? (E)

$0.08 \quad \mathbf{0 . 5 2}$

22. Do your family think you do things well at home? (E)

$0.37 \quad \mathbf{0 . 6 0}$

23. Do your family help you if you have a problem? (E)

$\mathbf{0 . 7 4} 0.33$

24. Do you think you are a help to your family? (E)

$0.38 \quad \mathbf{0 . 8 5}$

Peer dimension

25. Do other children like you for who you are? $(\mathrm{H})$

$\begin{array}{ll}\mathbf{0 . 8 3} & 0.47\end{array}$

26. Do other children make you feel happy? $(\mathrm{H})$

$\mathbf{0 . 4 8} 0.43$

27. Do you feel safe with other children? (H)

$0.47 \quad \mathbf{0 . 7 2}$

28 . Do you think other children care about you? $(\mathrm{H})$

$\mathbf{0 . 5 4} 0.48$

29. Do other children allow you to do the things you want to do? (E)

$0.39 \quad \mathbf{0 . 7 4}$

30. Do other children think you do things well when you play with them? (E) $\quad \begin{array}{ccc}\mathbf{0} & 0.54\end{array}$

31. Do other children help you if you have a problem? (E)

$0.37 \quad \mathbf{0 . 5 4}$

32. Do you think you are a help to other children? (E)

$0.35 \quad \mathbf{0 . 6 1}$

Bold values indicate the pattern of factor loadings for the constructs of hedonia and eudaimonia $H$ hedonia item, $E$ eudaimonia item, $F 1$ factor 1 hedonia, $F 2$ factor 2 eudaimonia 


\section{General Discussion}

Measuring child and adolescent wellbeing is a complex task, with many types of quantitative and qualitative approaches being used across high and low-income countries (e.g., Crivello et al., 2009; Ager et al., 2014). In this study we developed a conceptual framework for measuring children's eudaimonic and hedonic wellbeing based on humanistic and positive psychology (e.g., Deci \& Ryan, 2008; Ryan \& Deci, 2019) and social evolution theory (Tomasello \& Vaish, 2013). We investigated the ecological validity of the framework in focus group interviews with 19 children and 21 adults living in two rural communities. This work informed the concurrent development of a 32-item measure (the CAPSAW) during a longitudinal pilot study with 200 children (3-years-10-years). We tested the measure's validity in the last wave of the pilot study, and in the first wave of our main study with approximately 2000 children (4-years-13-years), living in 95 communities in rural Sierra Leone. Our qualitative investigation demonstrated the relevance of the social evolutionary framework to the lived experiences of children and adolescents in the local communities. Quantitative testing of the CAPSAW revealed that the measure had good dimensional validity, concurrent validity, and predictive validity. We discuss these results in turn.

\subsection{Functional Relevance of the Wellbeing Indicators}

We conceptualized that wellbeing emerges out of the core social functions necessary for human fecundity, fitness, and survival in social communities (Tomasello \& Vaish, 2013). In the qualitative investigation we explored which aspects of wellbeing emerged as most relevant to the local context (in line with Berry et al.'s (2008) discovery goal) by asking adults and children to describe wellbeing inductively and by coding coded their responses into a framework of indigenous perceptions on wellbeing. This framework comprised themes of children being cared for, children participating in the community, children accessing education, and children being clean and healthy. In the Young Lives qualitative investigations of child wellbeing in India, Vietnam, Peru and Ethiopia, children and adults stressed the importance of children being accepted and cared for, children attending school and children having physical health and good grooming (Crivello et al., 2009). Similarly, in Glozah (2015), Ghanaian adolescents described the importance of having good health, being able to go to school, sleeping well and avoiding malaria, receiving love and encouragement from others, and avoiding relational stress, as key to their wellbeing.

We then investigated participants' understanding of our assumed wellbeing indicators of hedonia (happiness, acceptance, care, and safety) and eudaimonia (competence, helping, autonomy, and resilience that we developed in our social evolutionary perspective. This activity was in line with Berry et al.'s (2008) cross-cultural psychology transport and test goal. All indicators were relevant to participants' lived experiences in their communities, and there was no evidence that participants struggled to comprehend any of the indicator concepts. The interviews were conducted by native Temne speakers, who had agreed the translation of the concepts beforehand with the researcher. Participants gave concrete examples of child wellbeing in their communities, when discussing each indicator.

The eight indicators used in our study have also emerged in other research in Africa on indigenous conceptualizations of wellbeing, as we illustrate below with quotes from those qualitative studies regarding autonomy ("to be free to be yourself", Wilson Fadiji et al., 2019, p. 18; "I want to be heard!... And be taken seriously" (Savahl et al., 2015, p. 760), 
competence ("being able to achieve my target", Wilson Fadiji et al., 2019, p. 18; "to feel good about yourself", Savahl et al., 2015, p. 759), care ("when I'm able to help others", Wilson Fadiji et al., 2019, p. 20), happiness ("wellbeing means happiness" Wilson Fadiji et al., 2019, p. 19), acceptance ("to be accepted with who you are", Savahl et al., 2015, p. 759; "my brother frightens me... if I am not serious and I do fail, he washes his hands and leave me", Wilson \& Somhlaba, 2016, p. 93), resilience/problem solving ("I don't find it hard to take certain decisions", Glozah, 2015, p. 5; "my mum... tells me the way and how to go about it", Glozah, 2015, p. 6), and safety ("Being safe! That's the most important!", Savahl et al., 2015, p. 753).

In the rural Sierra Leonian context, culturally informed forms of the eight indicators of eudaimonia and hedonia emerged in our study. These were safety from adult violence, adults supporting children's personal development, and children being psychologically well. Being safe from adult harm, including violence and abuse, also emerged as a theme in Savahl et al.'s (2015) qualitative study of South African children's perceptions of wellbeing. Adults supporting children was also a key theme in Glozah's (2015) study of Ghanaian adolescents, who discussed the importance of mothers and fathers 'encouraging' children and helping them to solve problems. Likewise, fearing being socially rejected and materially deprived by family was reported by Ghanaian adolescents in Wilson and Somhlaba (2016). Regarding psychological health, a recent investigation of the mental health of children in Sierra Leone found that children's psychological wellness has culturally specific manifestations, including being angry and destructive, sad and disruptive, sad and withdrawn, having excessive worry, and feeling abnormal fear (Thulin et al., 2020). More research on indigenous conceptualizations of children's mental health as a component of wellbeing, and on the social structures that support and prevent violence and inclusion as a means to wellbeing/illbeing, is warranted.

Another interesting finding in our study, was that participants did not immediately connect children's happiness or mental health to wellbeing, although in the second discussion with our participants children gave many examples of the things that made them feel good about themselves and feel happy (e.g., going to school). One interpretation is that happiness is an outcome of wellbeing, rather than an indicator of wellbeing, in the Sierra Leonian indigenous psychosocial system. However, happiness has emerged in other research in West Africa as important for wellbeing. Ghanaian adults have used the concept of happiness as a proxy for wellbeing (Wilson Fadiji et al., 2019), and the idea of enjoying life has emerged as central to wellbeing in South Africa (Savahl et al., 2015). Possibly in our study, there was a disconnect between the notion of an individual 'being well' (as used in our interviews) and 'wellbeing' as broader relational concept (e.g., White, 2015).

\subsection{Dimensional Validity}

The structure of the full 32-item measure used in the pilot study followed the anticipated distinction between hedonia and eudaimonia, with minor variations from this pattern. The global subscale most clearly differentiated between hedonic and eudaimonic wellbeing in the pilot study and main study data. This clarity might have occurred because the global subscale items were a-contextual, meaning that they did not link to any domain specific experience involving a key social group or cultural context. Accordingly, they were freer to load onto separate factors representing hedonia and eudaimonia. 
Across the global, peer, teacher, and family dimensions measured in the pilot study, and across the global and teacher dimensions measured in the main study, the items intended to measure eudaimonia were more likely to load onto both factors relating to eudaimonia and hedonia. This indicates that certain indicators of eudaimonia closely merged with feelings of happiness, care, acceptance, and safety. This is not entirely unexpected, as an overlap between hedonia and eudaimonia was also documented in Wilson Fadiji et al.'s (2019) qualitative study of Ghanaian adults, where out of 120 coded statements of what wellbeing meant to participants, $47 \%$ of statements were coded as hedonic wellbeing, $23 \%$ of cases were coded as eudaimonic wellbeing, and $30 \%$ of cases were coded as being relevant to both hedonia and eudaimonia. Clearly, it is not possible to entirely distinguish eudaimonia from hedonia. This perspective is commensurate with the approach to understanding individual psychological functioning as a relational complex dynamic system where motives, feelings, representations, and strivings are continually meshed in development (e.g., Overton, 2015).

\subsection{Concurrent and Predictive Validity}

Using correlational statistical techniques, we examined the concurrent and predictive associations between the teacher and personal wellbeing dimensions and children's motivation/persistence, gender, age and home language, using the main study sample for a more robust analysis. As anticipated, hedonia and eudaimonia in these dimensions were closely associated with children's motivation/persistence at school. This result fits well with conceptualizations that eudaimonia manifests as people's continual striving for need fulfilment and optimal psychosocial functioning (Deci \& Ryan, 2008), which requires people to be motivated and persist at tasks, such as doing well at school, that can fulfil important needs and help people develop themselves.

Regarding the predictive validity of the measure, females in this study typically reported higher personal wellbeing, although the associations were very small. Follow up analyzes of each item using paired-sample t-tests, found that the gender differences were most pronounced for feeling cared for $(\mathrm{t}=2.436, p<0.001)$, and helping others $(\mathrm{t}=2.742, p<0.001)$. Presuming that these differences were not simply a cause of reporting bias or methodological error, we can interpret the result as females being more immersed in the system of reciprocal helping that is vital in hypercooperative societies (Tomaselo \& Vaish, 2013), even at these young ages. This result also maps well onto ethnographic observations of rural Sierra Leonian communities (Devine et al., in press, b), that girls are more heavily acculturated into domestic helping roles from an early age, than boys are. In studies conducted in Ghana, Glozah and Pevalin (2017) found that girls reported better mental health than males on average, and Wilson and Somhlaba (2018) found that males had higher levels of hope than girls. However, also in Ghana, Cebotari et al. (2018) did not find any gender differences in children's life satisfaction or happiness. Evidently, cultural, cohort, and methodological differences need to be considered when interpreting findings on gender and wellbeing in the West African context.

In our study, being older was predictive of higher eudaimonia, which aligns with findings that children's ability for self-regulation, memory, language, and motor behavior increases with age (Goswami, 2019). As children's cognitive and physical competencies develop, they become better able to contribute in practical ways (e.g., through chores, farming) to the fitness and survival of their communities. Studies on child identity find that many children in middle childhood (ages 8-10-years) imagine themselves 
in different work roles, developing their career aspirations for adulthood (Watson \& McMahon, 2016). From a social evolutionary perspective, this serves the purpose of helping the child differentiate themselves from others, in a manner that can support broader societal functioning where reciprocal care is distributed across a diverse set of work roles in society (Côté \& Levin, 2015). Age differences in wellbeing have also been found in Ghanaian samples, with older children reporting lower levels of mental illness (Glozah \& Pevalin, 2017) and higher school enjoyment (Cebotari et al., 2018). However, also in Ghana, researchers have observed no age differences in children's life satisfaction or happiness (Cebotari et al., 2018), hope, emotional wellbeing, and life satisfaction (Wilson \& Somhlaba, 2018).

Finally, we observed differences in wellbeing by language spoken at home. This could indicate two things. First, there might be legitimate cultural difference in wellbeing (created by cultural differences in the speaker's local psychosocial systems) between the Temne, Krio and Kuranko speakers. Due to a lack of comparative research on these populations, we were unable to find published studies to help explain our results. Alternatively, there might be a cultural response bias in how the participants responded to the wellbeing items, which has been documented in other cross-cultural studies of subjective wellbeing measures (Lai et al., 2013).

\section{Limitations and Future Research Directions}

There are several strengths of the current research including that it was mixed methods and longitudinal, had a pilot study and a large sample for the main study, and ensured that the instruments and fieldwork were sensitively adapted and administered by a local Sierra Leonian research team. Furthermore, our empirical investigations were preceded by a review of measures of wellbeing. However, there were also limitations to the research.

First, because the evaluation pilot pre-test needed to take place as soon as we had access to the field, we were not able to conduct our qualitative focus groups on wellbeing until after we had tested the first set of quantitative items. Fortunately, because the pilot study was longitudinal, we were still able to evaluate and refine the measure according to the qualitative results.

A second limitation is that we were not able to test the full measure in the main study. This was due to inclusion of additional items and measures that we decided were important for understanding the processes and outcomes relating to the randomized controlled trial that the measure development study was a part of. In the trial baseline measurement used in this analysis, we measured only the personal and teacher dimensions. To extend our data on the measure, we have designed each of the three follow up waves to contain both the personal dimension and one of the other dimensions (peer, family, and a repeated measure of the teacher dimension). Ideally, we would have tested the full measure at each time point.

A third limitation is that in our quantitative tests we only examined the measure's functioning with children and adolescents aged 3-13-years. This excludes participants in midadolescence and late-adolescence. Although we designed the measure to be as transferrable as possible for children and adolescents of different ages, future research is needed to establish if the measure performs well with children older than 13-years. 
A fourth limitation is that our qualitative focus groups were cross-sectional. Repeating the wellbeing interview with the same sample across a longer time-period might have increased the validity and scope of our results. Therefore, our main suggestion for future measure development studies is to conduct a longitudinal, qualitative, exploratory investigation before any item development begins.

A final point to make is that the measure was developed and tested in rural Sierra Leone. Following Berry et al. (2008) it is possible to assume that core psychological phenomena such as hedonia and eudaimonia (represented by our eight indicators) exist across cultures, whilst being culturally relative in how they manifest in development. An important future research direction is to test the transferability of the CAPSAW measure with other indigenous populations, cross-culturally. Researchers can examine whether the factor structure can be identified cross-culturally using exploratory factor analysis, and whether there is factorial invariance across cultures and subgroups using confirmatory factor analysis (as has been done previously with studies of parenting socialization measures: Garcia et al., 2019), and whether the scores have an expected positive association with other similar indicators of positive development, such as self-esteem and life satisfaction, across cultures.

\section{Conclusions}

The Child and Adolescent Personal and Social Assessment of Wellbeing (CAPSAW) was developed and tested with a large sample of children in Sierra Leone. The qualitative and quantitative results support our conceptual framework based on social evolutionary theory, where we assumed children's wellbeing could be identified through their self-reported feelings of happiness, care, acceptance, safety (hedonia), autonomy, competence, helping others, and resilience (eudaimonia). A key finding from both studies was that children's lived experiences of these indicators of wellbeing was shaped by the sociocultural structure of their communities. We found many similarities between our results and those generated with different cultural samples in African contexts. Underpinning these similarities is the social evolutionary perspective of how hypercooperative societies promote human fecundity, fitness and survival through reciprocal helping which requires the development of individual competencies and community participation (Tomasello \& Vaish, 2013). Accordingly, our empirical investigation supports the position that child hedonic and eudaimonic wellbeing can have universally recognizable indicators, even though these indicators and their associations are also subject to cultural relativity (Berry et al., 2008).

Authors' Contributions Symonds-conceptualization, formal analysis, funding acquisition, investigation, methodology, project administration, supervision, validation, visualization, writing-original draft; Sloan-conceptualization, data curation, formal analysis, funding acquisition, investigation, methodology, project administration, supervision, writing — review and editing; Kearns_conceptualization, data curation, investigation, methodology, project administration; Devine-conceptualization, funding acquisition, project administration, supervision, writing - review and editing; Sugrue-conceptualization, funding acquisition, project administration, supervision, writing — review and editing; Suryanaryan-data curation, formal analysis, investigation; Capistrano_-data curation, formal analysis, writing—review and editing; Samonovawriting-review and editing.

Funding Open Access funding provided by the IReL Consortium. The research was funded by Concern Worldwide, through a grant awarded to Prof. Dympna Devine, Prof. Ciaran Sugrue, Associate Professor Jennifer Symonds, and Assistant Professor Seaneen Sloan; School of Education, University College Dublin. 
Data Availability A synthetic version of the data for replicating the wellbeing analysis can be requested by writing to Jennifer Symonds and Dympna Devine.

Code Availability The MPlus syntax can be requested by writing to Jennifer Symonds.

\section{Declarations}

Conflict of interest There are no conflicts of interest to report for this study.

Open Access This article is licensed under a Creative Commons Attribution 4.0 International License, which permits use, sharing, adaptation, distribution and reproduction in any medium or format, as long as you give appropriate credit to the original author(s) and the source, provide a link to the Creative Commons licence, and indicate if changes were made. The images or other third party material in this article are included in the article's Creative Commons licence, unless indicated otherwise in a credit line to the material. If material is not included in the article's Creative Commons licence and your intended use is not permitted by statutory regulation or exceeds the permitted use, you will need to obtain permission directly from the copyright holder. To view a copy of this licence, visit http://creativecommons.org/licenses/by/4.0/.

\section{References}

Ager, A., Robinson, S., \& Metzler, J. (2014). Methodologies and tools for measuring the mental health and psychosocial wellbeing of children in humanitarian contexts. New York: Columbia University, Columbia Group for Children in Adversity \& Child Protection in Crisis (CPC) Network. http://www. cpcnetwork.org/wp-content/uploads/2015/06/Measuring-Child-MHPSS-in-Emergencies_CU_Mappi ng-Report_March-2014.pdf

Bentler, P. M. (1990). Comparative fit indexes in structural models. Psychological Bulletin, 107(2), 238246. https://doi.org/10.1037/0033-2909.107.2.238

Berry, J. E., Poortinga, Y. H., Segall, M. H., \& Dasen, P. R. (2008). Cross-cultural psychology: Research and applications. Cambridge University Press.

Cebotari, V., Mazzucato, V., \& Appiah, E. (2018). A longitudinal analysis of well-being of ghanaian children in transnational families. Child Development, 89(5), 1768-1785. https://doi.org/10.1111/ cdev.12879

Chirkov, V., Ryan, R. M., Kim, Y., \& Kaplan, U. (2003). Differentiating autonomy from individualism and independence: A self-determination theory perspective on internalization of cultural orientations and well-being. Journal of Personality and Social Psychology, 84(1), 97-110. https://doi.org/ 10.1037/0022-3514.84.1.97

Clarke, V., Braun, V., \& Hayfield, N. (2015). Thematic analysis. In J. A. SMit (Ed.), Qualitative psychology: A practical guide to research methods (3rd ed., pp. 222-248). SAGE Publications Inc.

Côté, J. E., \& Levine, C. (2015). Identity formation, youth, and development: A simplified approach. Psychology Press.

Crivello, G., Camfield, L., \& Woodhead, M. (2009). How can children tell us about their wellbeing? Exploring the potential of participatory research approaches within young lives. Social Indicators Research, 90(1), 51-72. https://doi.org/10.1007/s11205-008-9312-x

Cummings, R. A., \& Lau, A. L. D. (2005). Personal wellbeing index - school children (PWI-SC). 3rd Edition. Manual. Melbourne, Victoria, Australia: School of Psychology, Deakin University. http:// www.acqol.com.au/uploads/pwi-sc/pwi-sc-english.pdf

David, S., Boniwell, I., \& Conley Ayers, A. (Eds.). (2013). The Oxford handbook of happiness. Oxford University Press.

Deci, E. L., \& Ryan, R. M. (2008). Hedonia, eudaimonia, and well-being: An introduction. Journal of Happiness Studies, 9(1), 1-11. https://doi.org/10.1007/s10902-006-9018-1

Devine, D., Bolotta, G., Samonova, E., Sugrue, C., Sloan, S., Symonds, J., \& Capistrano, D. (2021). Becoming 'brilliant': Generationing education and development in rural Sierra Leone. Childhood, 28(2), 262-278. https://doi.org/10.1177/0907568220981159

Devine, D., Samonova, E., Bolotta, G., Sugrue, C., Sloan, S., Symonds, J. E., Capistrano, D., \& Crean, M. (in press a). Gendering childhood(s) and engagement with schooling in rural Sierra Leone. Compare: A Journal of Comparative and International Education. 
Devine, D., Bolotta, G., Samonova, E., Sloan, S., Sugrue, C., Symonds, J. E., \& Capistrano, D. (in press b). Becoming brilliant: Generationing education and development in rural Sierra Leone. Childhood.

Diener, E., Emmons, R. A., Larsen, R. J., \& Griffin, S. (1985). The satisfaction with life scale. Journal of Personality Assessment, 49(1), 71-75. https://doi.org/10.1207/s15327752jpa4901_13

Garcia, F., Serra, E., Garcia, O. F., Martinez, I., \& Cruise, E. (2019). A third emerging stage for the current digital society? Optimal parenting styles in Spain, the United States, Germany, and Brazil. International Journal of Environmental Research and Public Health, 16(2333), 1-20. https://doi. org/10.3390/ijerph16132333

Glozah, F. N. (2015). Exploring Ghanaian adolescents' meaning of health and wellbeing: A psychosocial perspective. International Journal of Qualitative Studies on Health and Well-Being, 10(1), 26370. https://doi.org/10.3402/qhw.v10.26370

Glozah, F. N., \& Pevalin, D. J. (2017). Association between psychosomatic health symptoms and common mental illness in Ghanaian adolescents: Age and gender as potential moderators. Journal of Health Psychology, 22(11), 1376-1386. https://doi.org/10.1177/1359105316628736

Goodman, R., Meltzer, H., \& Bailey, V. (1998). The strengths and difficulties questionnaire: A pilot study on the validity of the self-report version. European Child \& Adolescent Psychiatry, 7(3), 125-130. https://doi.org/10.1007/s007870050057

Goswami, U. (2019). Cognitive development and cognitive neuroscience: The learning brain (2nd ed.). Routledge.

Harter, S. (2012). Self-perception profile for children: Manual and questionnaires. University of Denver.

Henrich, J., \& Muthukrishna, M. (2021). The origins and psychology of human cooperation. Annual Review of Psychology, 72(1), 207-240. https://doi.org/10.1146/annurev-psych-081920-042106

Hu, L. T., \& Bentler, P. M. (1999). Cutoff criteria for fit indexes in covariance structure analysis: Conventional criteria versus new alternatives. Structural Equation Modeling: A Multidisciplinary Journal, 6(1), 1-55. https://doi.org/10.1080/10705519909540118

Kingdon, G. G., \& Knight, J. (2006). Subjective well-being poverty vs. Income poverty and capabilities poverty? The Journal of Development Studies, 42(7), 1199-1224. https://doi.org/10.1080/00220 380600884167

Lai, L. C. H., Cummins, R. A., \& Lau, A. L. D. (2013). Cross-cultural difference in subjective wellbeing: Cultural response bias as an explanation. Social Indicators Research, 114(2), 607-619. https:// doi.org/10.1007/s11205-012-0164-Z

Lyubomirsky, S., \& Lepper, H. S. (1999). A measure of subjective happiness: Preliminary reliability and construct validation. Social Indicators Research, 46(2), 137-155. https://doi.org/10.1023/A:10068 24100041

Marsh, H. W., Craven, R., \& Debus, R. (1998). Structure, stability, and development of young children's self-concepts: A multicohort-multioccasion study. Child Development, 69(4), 1030-1053. https:// doi.org/10.2307/1132361

Maslow, A. H. (1943). A theory of human motivation. Psychological Review, 50(4), 370-396. https:// doi.org/10.1037/h0054346

McLellan, R., \& Steward, S. (2015). Measuring children and young people's wellbeing in the school context. Cambridge Journal of Education, 45(3), 307-332. https://doi.org/10.1080/0305764X. 2014.889659

Overton, W. F. (2015). Processes, relations, and relational developmental systems. In R. M. Lerner (Ed.), Handbook of child psychology and developmental science (pp. 9-62). Wiley.

Patrick, D. L., Edwards, T. C., \& Topolski, T. D. (2002). Adolescent quality of life, Part II: Initial validation of a new instrument. Journal of Adolescence, 25(3), 287-300. https://doi.org/10.1006/jado. 2002.0471

Queiroz, P., Garcia, O. F., Garcia, F., Zacares, J. J., \& Camino, C. (2020). Self and nature: Parental socialization, self-esteem, and environmental values in Spanish adolescents. International Journal of Environmental Research and Public Health, 17(3732), 1-13. https://doi.org/10.3390/ijerph1710 3732

Rees, G., \& Main, G. (Eds.). (2015). Children's views on their lives and well-being in 15 countries: An initial report on the Children's Worlds survey, 2013-14. Children's Worlds Project (ISCWeB). https://isciweb.org/

Reeve, J. (2015). Growth motivation and positive psychology. In Understanding motivation and emotion (6th edn., pp. 431-465). Wiley

Rodrigues, A. M. M., \& Kokko, H. (2016). Models of social evolution: Can we do better to predict "who helps whom to achieve what"? Philosophical Transactions of the Royal Society B: Biological Sciences. https://doi.org/10.1098/rstb.2015.0088 
Ryan, R. M., \& Deci, E. L. (2019). Chapter four-brick by brick: The origins, development, and future of self-determination theory. In A. J. Elliot (Ed.), Advances in motivation science (Vol. 6, pp. 111156). Elsevier. https://doi.org/10.1016/bs.adms.2019.01.001

Savahl, S., Adams, S., Florence, M., Casas, F., Mpilo, M., Louise Sinclair, D., \& Manuel, D. (2020). Afrikaans adaptation of the children's hope scale: Validation and measurement invariance. Cogent Psychology, 7(1), 1-16. https://doi.org/10.1080/23311908.2020.1853010

Savahl, S., Casas, F., \& Adams, S. (2017). Children's subjective well-being: Multi-group analysis among a sample of children from two socio-economic status groups in the Western Cape, South Africa. Child Indicators Research, 10(2), 473-488. https://doi.org/10.1007/s12187-016-9392-0

Savahl, S., Malcolm, C., Slembrouk, S., Adams, S., Willenberg, I. A., \& September, R. (2015). Discourses on well-being. Child Indicators Research, 8(4), 747-766. https://doi.org/10.1007/ s12187-014-9272-4

Scales, P. C. (2011). Youth developmental assets in global perspective: Results from international adaptations of the developmental assets profile. Child Indicators Research, 4(4), 619-645. https://doi. org/10.1007/s 12187-011-9112-8

Scheier, M. F., Carver, C. S., \& Bridges, M. W. (1994). Distinguishing optimism from neuroticism (and trait anxiety, self-mastery, and self-esteem): A reevaluation of the Life Orientation Test. Journal of Personality and Social Psychology, 67, 1063-1078. https://doi.org/10.1037/0022-3514.67.6.1063

Seligson, J. L., Huebner, E. S., \& Valois, R. F. (2003). Preliminary validation of the Brief Multidimensional Students' Life Satisfaction Scale (BMSLSS). Social Indicators Research, 61(2), 121-145. https://doi. org/10.1023/A:1021326822957

Senefeld, S., Strasser, S., \& Campbell, J. (2009). Orphans and vulnerable children wellbeing tool. Catholic Relief Services. https://www.crs.org/sites/default/files/tools-research/orphans-vulnerable-child ren-wellbeing-tool-users-guide.pdf

Snyder, C. R., Hoza, B., Pelham, W. E., Rapoff, M., Ware, L., Danovsky, M., Highberger, L., Ribinstein, H., \& Stahl, K. J. (1997). The development and validation of the Children's Hope Scale. Journal of Pediatric Psychology, 22(3), 399-421. https://doi.org/10.1093/jpepsy/22.3.399

Steger, M. F., Frazier, P., Kaler, M., \& Oishi, S. (2006). The meaning in life questionnaire: Assessing the presence of and search for meaning in life. Journal of Counseling Psychology, 53(1), 80-93. https://doi.org/10.1037/0022-0167.53.1.80

Steiger, J. H. (1990). Structural model evaluation and modification: An interval estimation approach. Multivariate Behavioral Research, 25(2), 173-180. https://doi.org/10.1207/s15327906mbr2502_4

Thulin, E. J., McLean, K. E., Sevalie, S., Akinsulure-Smith, A. M., \& Betancourt, T. S. (2020). Mental health problems among children in Sierra Leone: Assessing cultural concepts of distress. Transcultural Psychiatry, 1-18. https://doi.org/10.1177/1363461520916695

Tomasello, M., \& Vaish, A. (2013). Origins of human cooperation and morality. Annual Review of Psychology, 64(1), 231-255. https://doi.org/10.1146/annurev-psych-113011-143812

Tsang, K. L. V., Wong, P. Y. H., \& Lo, S. K. (2012). Assessing psychosocial well-being of adolescents: A systematic review of measuring instruments. Child: Care, Health and Development, 38(5), 629646. https://doi.org/10.1111/j.1365-2214.2011.01355.x

Ungar, M., \& Liebenberg, L. (2011). Assessing resilience across cultures using mixed methods: Construction of the Child and Youth Resilience Measure. Journal of Mixed Methods Research, 5(2), 126-149. https://doi.org/10.1177/1558689811400607

van Manen, M., \& Levering, B. (1996). Childhood's secrets intimacy, privacy, and the self reconsidered. Teachers College Press.

Varni, J. W., Limbers, C. A., \& Burwinkle, T. M. (2007). How young can children reliably and validly selfreport their health-related quality of life?: An analysis of 8,591 children across age subgroups with the PedsQL ${ }^{\text {TM }}$ 4.0 Generic Core Scales. Health and Quality of Life Outcomes, 5(1), 1-13. https://doi.org/ 10.1186/1477-7525-5-1

Vitters $\emptyset$, J. (2013). Functional wellbeing: Happiness as feelings, evaluations, and functioning. In S. David, I. Boniwell, \& A. C. Ayers (Eds.), The Oxford handbook of happiness (pp. 227-246). Oxford University Press.

Wang, M.-T., Fredricks, J., Ye, F., Hofkens, T., \& Linn, J. S. (2017). Conceptualization and assessment of adolescents' engagement and disengagement in school: A multidimensional school engagement scale. European Journal of Psychological Assessment. https://doi.org/10.1027/1015-5759/a000431

Watson, M., \& McMahon, M. (Eds.). (2016). Career exploration and development in childhood: Perspectives from theory, practice and research. Routledge.

White, S. C. (2015). Relational wellbeing: A theoretical and operational approach. Bath Papers in International Development and Wellbeing, No. 43. University of Bath, Centre for Development Studies (CDS). https://www.econstor.eu/bitstream/10419/128138/1/bpd43.pdf 
White, S. C., \& Jha, S. (2018). Towards an interdisciplinary approach to wellbeing: Life histories and selfdetermination theory in rural Zambia. Social Science \& Medicine, 212, 153-160. https://doi.org/10. 1016/j.socscimed.2018.07.026

Wilson, A., \& Somhlaba, N. (2016). Psychological well-being in a context of adversity: Ghanaian adolescents' experiences of hope and life satisfaction. Africa Today, 63(1), 85-103. https://doi.org/10.2979/ africatoday.63.1.0085

Wilson, A., \& Somhlaba, N. Z. (2018). Gender, age, religion and positive mental health among adolescents in a Ghanaian socio-cultural context. Child Indicators Research, 11(4), 1131-1158. https://doi.org/10. 1007/s12187-017-9495-2

Wilson Fadiji, A., Meiring, L., \& Wissing, M. P. (2019). Understanding well-being in the Ghanaian context: Linkages between lay conceptions of well-being and measures of hedonic and eudaimonic well-being. Applied Research in Quality of Life. https://doi.org/10.1007/s11482-019-09777-2

Yates, A. (1987). Multivariate exploratory data analysis: A perspective on exploratory factor analysis. Suny Press

Young Lives. (2009). Child Questionnaire for 7.5-8.5 Year Olds (Younger Cohort). Oxford, UK: Ethiopian Development Research Institute (Ethiopia), University of Oxford (UK). https://www.younglives.org. uk/content/round-3-questionnaires

Publisher's Note Springer Nature remains neutral with regard to jurisdictional claims in published maps and institutional affiliations. 